\title{
Checking is Believing: Event-Aware Program Anomaly Detection in Cyber-Physical Systems
}

\author{
Long Cheng, Member, IEEE, Ke Tian, Danfeng (Daphne) Yao, Senior Member, IEEE, \\ Lui Sha, Fellow, IEEE, and Raheem A. Beyah, Senior Member, IEEE
}

\begin{abstract}
Securing cyber-physical systems (CPS) against malicious attacks is of paramount importance because these attacks may cause irreparable damages to physical systems. Recent studies have revealed that control programs running on CPS devices suffer from both control-oriented attacks (e.g., code-injection or code-reuse attacks) and data-oriented attacks (e.g., non-control data attacks). Unfortunately, existing detection mechanisms are insufficient to detect runtime data-oriented exploits, due to the lack of runtime execution semantics checking. In this work, we propose Orpheus, a new security methodology for defending against data-oriented attacks by enforcing cyber-physical execution semantics. We first present a general method for reasoning cyber-physical execution semantics of a control program (i.e., causal dependencies between the physical context/event and program control flows), including the event identification and dependence analysis. As an instantiation of Orpheus, we then present a new program behavior model, i.e., the event-aware finite-state automaton (eFSA). eFSA takes advantage of the event-driven nature of CPS control programs and incorporates event checking in anomaly detection. It detects data-oriented exploits if a specific physical event is missing along with the corresponding event dependent state transition. We evaluate our prototype's performance by conducting case studies under data-oriented attacks. Results show that eFSA can successfully detect different runtime attacks. Our prototype on Raspberry $\mathrm{Pi}$ incurs a low overhead, taking $0.0001 \mathrm{~s}$ for each state transition integrity checking, and $0.063 \mathrm{~s} \sim 0.211 \mathrm{~s}$ for the cyber-physical contextual consistency checking.
\end{abstract}

Index Terms-Cyber-physical systems, Data-oriented attacks, Program anomaly detection, Cyber-physical execution semantics.

\section{INTRODUCTION}

Q YBER-physical systems (CPS) consist of a tightly coupled integration of computational elements and physical components. The computational elements rely on sensors to monitor the physical environment and make control decisions to affect physical processes with feedback loops [2]. These systems are widely used to operate critical infrastructure assets, such as electric power grid, oil and natural gas distribution, industry automation, medical devices, automobile systems, and air traffic control [3]. In the industrial control domain, CPSs are instantiated as the Industrial Control Systems (ICS), Distributed Control Systems (DCS), or Supervisory Control and Data Acquisition (SCADA) systems [4]. Though CPS and IoT (Internet of Things) are defined with different emphasis and have no standard definitions agreed upon by the research community, they have significant overlaps. In general, CPS emphasizes the tightly coupled integration of computational components and physical world. While IoT has an emphasis on the connection of things with networks. If an IoT system interacts with the physical world via sensors/actuators, we can also classify it as a CPS [5].

The tight coupling with physical space of CPS brings new security and safety challenges. Control programs running on CPS devices monitor physical environments by taking sensory data as input and send control signals that affect physical systems [6]. They are critical to the proper operations of CPS, as anomalous program behaviors can have serious consequence, or even cause devastating damages to physical systems [7]. For example, the Stuxnet [8] attack allows hackers to compromise the control system of a nuclear power plant and manipulate real-world equipment such as centrifuge rotor speeds, which can be very dangerous.

A preliminary version of this work appeared in $[1]$.
According to ICS-CERT's report [9], there have been continuously increasing number of cyber attacks targeting critical infrastructure. Therefore, securing CPS against malicious attacks becomes of paramount importance in the prevention of potential damages to physical systems.

Recent studies [7], [10], [11], [12], [13], [14] have shown that control programs suffer from a variety of runtime software exploits. These attacks can be broadly classified into two categories:

- Control-oriented attacks exploit memory corruption vulnerabilities to divert a program's control flows, e.g., malicious code injection [15] or code reuse attacks [11]. Control-oriented attacks in conventional cyber systems (i.e., without cyber-physical interactions) have been well studied [16]. It is possible that existing detection approaches [17], [18], [19], [20], [21], [22] are extended to defend against control-oriented attacks in CPS.

- Data-oriented attacks manipulate program's internal data variables without violating its control-flow integrity (CFI), e.g., non-control data attacks [23], control-flow bending [22], dataoriented programming [24]. Data-oriented attacks are much more stealthy than attacks against control flows. Because existing CFI-based solutions are rendered defenseless under dataoriented attacks, such threats are particularly alarming. We mainly focus on runtime software exploits, and thus sensor data spoofing attacks [25], |26] in the physical domain are out of the scope in this work.

Since many control decisions are made based on particular values of data variables in control programs [7], data-oriented attacks could potentially cause serious harm to physical systems in a stealthy way. We further categorize data-oriented attacks against control programs into two types. i) Attacks on control branch, 
which corrupt critical decision making variables at runtime to execute a valid-yet-unexpected control-flow path (e.g., allowing liquid to flow into a tank despite it is full [27] or preventing a blast furnace from being shut down properly as in the recent German steel mill attack [28]). ii) Attacks on control intensity, which corrupt sensor data variables to manipulate the amount of control operations, e.g., affecting the number of loop iterations to dispense too much drug [7]).

In many instances, CPS can be modeled as event-driven control systems [29], [30]. We refer to events as occurrences of interest that come through the cyber-physical observation process or emitted by other entities (e.g., the remote controller), and trigger the execution of corresponding control actions. Defending against CPS data-oriented attacks is challenging due to the following reasons. First, data-oriented exploits can achieve attack goals without incurring illegal control flows, thus providing opportunities for attackers to evade all control flow integrity based detections [24]. Second, CPS programs normally rely on external sensor events to make control decisions. This physical event-driven nature makes it difficult to predict runtime program behaviors in CPS. Hence, an anomaly detection system needs to check the runtime integrity of program behaviors from both cyber and physical domains. Unfortunately, there exist very few defenses [7], [31] and they are ineffective to prevent both attack types due to the lack of runtime execution semantics checking.

Goals and Contributions. In this paper, we focus on a new type of runtime attacks that result in inconsistencies between the physical context/event and program execution, where executed control flow paths do not correspond to the observed events. These attacks do not necessarily violate any control flow integrity, so existing techniques based on control flow checking are not effective. We point out the need for an event-aware controlprogram anomaly detection, which reasons about program behaviors with respect to cyber-physical interactions, e.g., whether or not to open a valve is based on the current ground truth water level of a tank [27]. None of existing program anomaly detection solutions [16] has the event-aware detection ability. They cannot detect attacks that cause inconsistencies between program control flow paths and the physical environments.

We address the problem of securing control programs against data-oriented attacks, through enforcing the execution semantics of control programs in the cyber-physical domain. Specifically, our program anomaly detection enforces the consistency among control decisions, values of data variables in control programs, and the physical environments. Our main technical contributions are summarized as follows.

- We describe a new security methodology, named Orpheus, that leverages the event-driven nature in characterizing CPS control program behaviors. We present a general method for reasoning cyber-physical execution semantics of a control program, including the event identification and event dependence analysi 1

- As an instantiation of Orpheus, we present a new event-aware finite-state automaton ( $e$ FSA) model to detect anomalous control program behaviors particularly caused by data-oriented attacks in CPS. By enforcing runtime cyber-physical execution semantics, $e$ FSA detects subtle data-oriented exploits when physical event are inconsistent with the corresponding event-dependent state transitions. While our exposition of Orpheus is on an FSA model at the system call level, the design paradigm of Orpheus

1. Accompanying materials of this work are available at goo.gl/Wkrdzz can be used to augment many existing program behavior models, such as the n-gram model [32] or HMM model [33].

- We implement a proof-of-concept prototype on Raspberry Pi platforms, which have emerged as popular devices for building CPS applications [7], [34], [35]. Our prototype features: i) A gray-box FSA model that examines the return addresses on the stack when system calls are made, and thus significantly increases the bar for constructing evasive mimicry attacks. ii) An LLVM-based event dependence analysis tool to extract event properties from programs and correlate the physical event with runtime program behaviors, which we refer to as cyberphysical execution semantics. iii) A near-real-time anomaly detector using named pipes, with both local and distributed event verifiers to assess the physical context.

- We conduct a thorough evaluation of eFSA's performance through real-world CPS case studies. Results show that our approach can successfully detect different runtime data-oriented attacks reproduced in our experiments. Our prototype of the runtime anomaly detector takes $\sim 0.0001$ s to check each state transition in $e$ FSA model, $\sim 0.063$ s for the local event verification, and $\sim 0.211 \mathrm{~s}$ for the distributed event verification.

\section{BACKground ANd ATtACK MOdEL}

In this section, we introduce the CPS background, and describe the attack model and assumptions of this work. We use examples to illustrate our new detection capabilities.

\subsection{CPS Background}

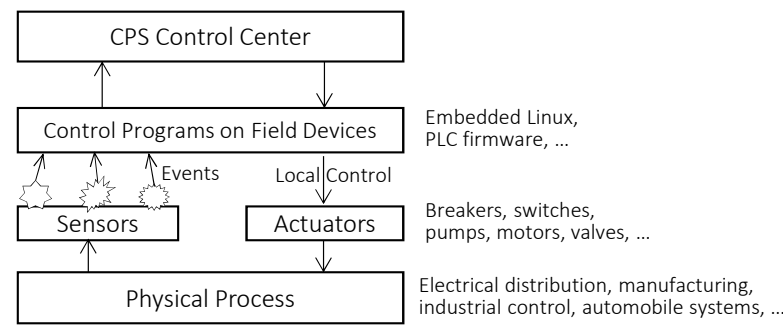

Fig. 1: An abstract view of the event-driven CPS architecture. CPS is exposed with a large attack surface and attacks can be launched across all components in the system.

Fig. 1 shows an abstract view of the CPS system architecture, which is also in line with the architecture of modern Industrial Control Systems (ICS). In industrial control domain, the control program is often referred to as control logic, and the firmware on PLC (i.e., field device) acts as a kind of operating system [36]. In general, it is composed of the following components: 1) a physical process (e.g., industrial plant or smart home); 2) sensors that measure the physical environment; 3) actuators that trigger physical changes in response to control commands sent by the control program; 4) control programs running on embedded devices that supervise and control physical processes by taking sensory data as input and making local control decisions; 5) a remote control server (which is optional), letting users remotely monitor and control the physical process. CPS communicates with the physical process through sensors and actuators, where physical environments are sensed and events (e.g., coming from the environment or emitted by other entities) are detected, and then actuation tasks are executed through a set of actuators.

Embedded devices (a.k.a. field devices) in CPS are situated in the field, where their operating systems are typically embedded 
Linux/Windows variants [37] or PLC firmware [36]. Traditionally, embedded control systems were not considered prominent attack targets due to their isolation from potential attack sources. However, the historical isolation has begun to break down as more and more embedded devices are connected to business networks and the Internet in the trend of IoT, making CPS control programs increasingly vulnerable [37].

\subsection{Attack Model and Assumptions}

In this paper, we make the following security assumptions:

- Capabilities of the adversary. We assume that the adversary has successfully authenticated CPS field devices (or the control server) under her control to the local network, and is able to launch runtime software exploits which may be unknown or known but unpatched at the time of intrusion. We are not concerned how attackers gained entry into the devices and launch different attacks, but focus on uncovering abnormal program execution behaviors after that [21]. This is a typical assumption in existing anomaly detection works.

- CPS platform. We assume the initial state (i.e., the training stage) of the application is trustworthy, which is a general requirement of most behavior-based intrusion detection systems [31]. We also assume the runtime monitoring module (running on the host) is trusted and cannot be disabled or modified. This assumption is reasonable because it can be achieved by isolating the anomaly detector (monitoring module) from the untrusted target program with hardware security support such as Intel's TrustLite or ARM's TrustZone [7]. At the time of detection, the user space is partially or fully compromised, but the operating system space has not been fully penetrated yet, and thus it is still trusted [10].

- Our focus. We focus our investigation on runtime software exploits, and thus sensor data spoofing attacks in the physical domain [26] are out of the scope. We assume sensor measurements are trustable. We limit our attention to data-oriented attacks that involve changes of system call usage. Other datarelated attacks that do not impact observable program behavior patterns (e.g., modification of non-decision making variables) are beyond the scope of this work. System call can be used as an ideal signal for detecting potential intrusions, since a compromised program can generally cause damage to the victim system only by exploiting system calls [38]. Despite system call based monitoring is widely used for detecting compromised programs, we aim at developing a CPS-specific anomaly detection system by augmenting an existing program behavior model with physical context awareness.

\subsection{New Detection Capabilities}

Our new detection capability is detecting data-oriented attacks in CPS control programs, including hijacked for/while-loops or conditional branches. These stealthy attacks alter the underlying control program's behaviors without tampering control-flow graphs (CFGs). We illustrate our new detection capabilities using a smart syringe pump as an example ${ }^{2}$ The control program reads humidity sensor values as well as takes remote user commands, and translates the input values/commands into control signals to its actuator. Partial code is shown in Listing 1 Suppose a stack buffer overflow vulnerability exists in the recvRemoteCommand ()

2. https://hackaday.io/project/1838-open-syringe-pump function (line 3). When the vulnerable function is invoked, an attacker is able to corrupt the sensor variables (e.g., pressure, temperature, and humidity) in the program. Our approach reasons about control programs' behaviors w.r.t physical environments, and is able to detect the following attacks:

- Attacking control branch. An attack affecting the code in lines 5 and 7 of Listing 1 may trigger push-syringe or pull-syringe regardless of physical events or remote requests. It corrupts control variables that result in event function push_event or pull_event returning True. Such an attack leads to unintended but valid control flows.

- Attacking control intensity. An attack may directly or indirectly corrupt a local state variable (e.g., steps in line 18 of Listing (1) that controls the amount of liquid to dispense by the pump. Such an attack may cause the syringe to overpump than what is necessary for the physical system. Range-based anomaly detection would not work, as the overwritten variable may still be within the permitted range but incompatible with the current physical context. Such an attack (i.e., manipulating the control loop iterations) does not violate the program's CFG either.

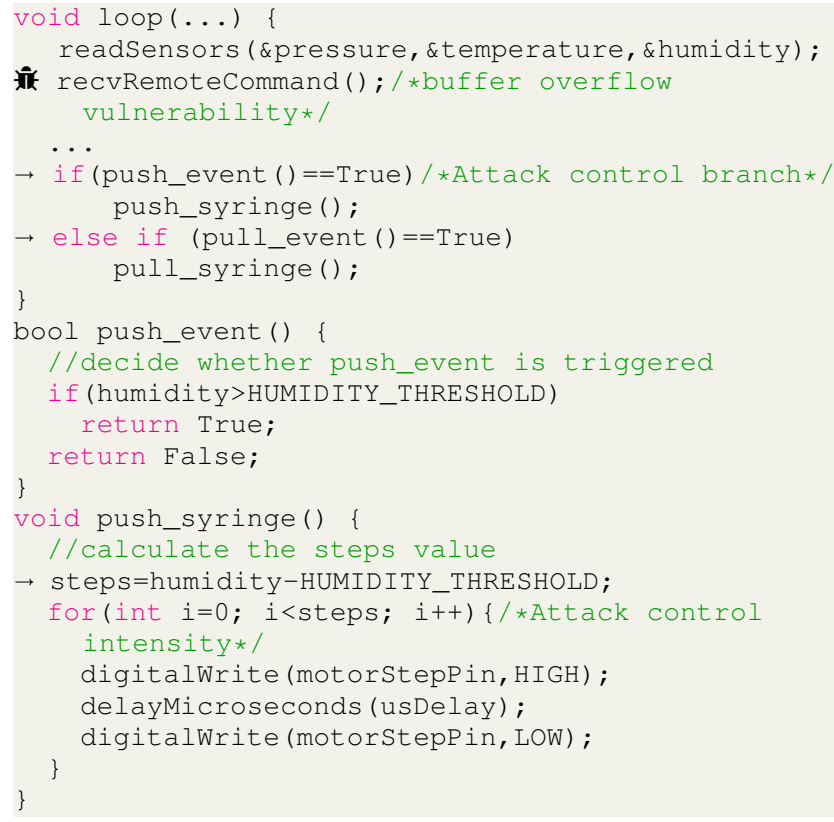

Listing 1: Examples of data-oriented attacks in a simplified smart syringe pump application. An attacker could purposely (a) trigger control actions by manipulating the return value of push_event or pull_event, and (b) manipulate the number of loop iterations in push-syringe without violating the control program's CFG.

Existing solutions cannot detect these attacks, as the detection does not incorporate events and cannot reason about program behaviors w.r.t. physical environments. C-FLAT [7], which is based on the attestation of control flows and a finite number of permitted execution patterns, cannot fully detect these attacks. Similarly, recent frequency- and co-occurrence-based anomaly detection approaches (e.g., global trace analysis [39] and system call frequency distribution (SCFD) [31]) cannot detect such either type of attacks, as their analyses do not model runtime cyberphysical context dependencies. 


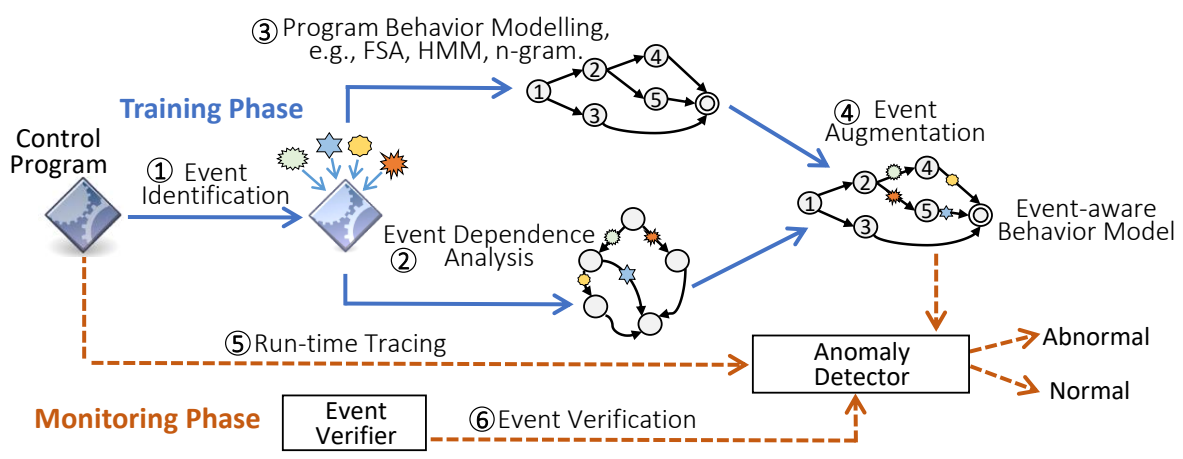

Fig. 2: Workflow of Orpheus event-aware anomaly detection framework, which augments an existing program behavior model with cyber-physical contextual integrity.

\subsection{Definition of Events}

Without loss of generality, we define two types of events in control programs: binary events and control-intensity events. In this work, the physical context refers to these physical events that trigger a particular execution path in a CPS program.

- Binary events return either True or False, which are defined in terms of pre-specified status changes of physical environments and provide notifications to the control program (e.g., push_event or pull_event in Listing 1]. Note that though sensor values such as temperature or humidity have continuous attributes that would lead to a large input space, binary events have a binary outcome which indicates a pre-specified status change is triggered. Such events are commonly pre-defined and used in CPS/IoT's trigger-action programming ("if, then") model [30], [40].

- Control-intensity events correspond to the sensor-driven control actions within a for/while loop, e.g., sensor values affect the amount of control operations of push-syringe in Listing 1 We consider each loop iteration as a discrete event. It is challenging to identify control-intensity events since they are not explicitly declared in control programs. We present a general event identification method in Sec.4.1

\section{Orpheus Anomaly Detection Framework}

\subsection{Motivation}

Runtime software attacks by exploiting memory corruption vulnerabilities constitute a major attack vector against CPS [41] [42]. This is because, low-level memory-unsafe languages (e.g., $\mathrm{C} / \mathrm{C}++)$ are widely used in embedded systems for speed performance purposes. As launching control-oriented attacks become increasingly difficult due to many deployed defenses against control-flow hijacking, data-oriented attacks are considered an appealing attack technique for system compromise.

Data-oriented attacks can purposely change the underlying CPS program behaviors and drive the system to unexpected states in a stealthy way, and thus posing a serious security threat to CPS. From the example in Listing 1] we observe that runtime control flows of CPS program are dependent on the external physical context. A data-oriented attack could lead to an inconsistency between the physical context and program control flow. This motivates us to leverage the intrinsic physical context dependency in CPS control programs as a channel to detect anomalous program behavior in CPS. Our key idea is to enforce physical context constraints over existing program behavior models, and check the consistency between runtime program behavior and external execution semantics.

\subsection{Design Overview}

Fig. 2 shows the workflow of Orpheus event-aware anomaly detection framework, which is a learning-based program anomaly detection and composed of two stages: training/learning (where program behavior models are built based on normal program traces) and monitoring/testing (where a new trace is compared against the model built in the training phase). In particular, to capture the cyber-physical context dependency of control programs, the training stage in Orpheus encompasses both static program analysis and dynamic profiling.

There are four main steps in the training phase. In step (1) (Sec. 4.1, Orpheus identifies both binary events and controlintensity events involved in the control program. In step (2) (Sec. 4.2), it performs the program dependency analysis to generate event-annotated CFG, which identifies the instructions/statements associated with binary events, and control-intensity loops associated with control-intensity events. In step (3) (Sec. 5.1), Orpheus constructs the normal program behavior model either based on static analysis or dynamic profiling, which we refer to as a basic program behavior model in Orpheus. The next step (4) (Sec. 5.2 is important. It augments the basic model with event constraints and obtains the event-aware program behavior model.

Steps (5) and (6) are the monitoring phase (Sec. 6). In step (5), the anomaly detector (which can be located in the secure world in ARM TrustZone to provide a trusted execution environment for trace collection [7] [43]) monitors the program's execution and collects runtime traces. The basic program behavior model normally aims at detecting control-oriented attacks. Our main contribution lies in the event awareness enhancement on top of the basic model. In the monitoring phase, whenever an eventdependent control-flow path is encountered in step (6), the event verifier checks the consistency between runtime behavior and program execution semantics, e.g., whether a specific physical event associated with an event-dependent control-flow path is observed in the physical domain. An anomaly is marked if there exists any deviation from the normal behavior model, or a mismatch between the physical context and program control-flow path.

\subsection{Program Behavior Model Choices}

Program behavior modeling has been an active research topic over the past decade and various models have been proposed for legacy applications [16]. Existing models can be classified 
into two categories: i) local model (e.g., n-gram model [32], hidden markov model (HMM) based approach [33], finite-state automaton (FSA) model [44]); and ii) long-range model (e.g., frequency distribution based models [31], [39], [45]). Local anomaly detection inspects short-range segments of program execution traces to detect anomalies such as control-flow violations. Longrange anomaly detection examines longer system behaviors (e.g., a complete program behavior instance) than the local anomaly detection, which can detect frequency anomalies. Among these models, system-call based monitoring is widely used for detecting compromised programs, in comparison to library/function-calls.

$\mathrm{N}$-gram based model defines the normal program behavior for a process by using short sequences of system-calls. In the training phase, it builds a n-gram database by sliding a window of length $n$ over the system-call traces of normal program executions. An anomaly is detected if a new n-gram is observed in the testing sequence (i.e., test for membership in the database). Although short-range ordering of system-calls have a high probability of being perturbed when abnormal activities occur, it is vulnerable to mimicry attacks [46]. An attacker may insert a malicious code, issuing system-calls accepted by a normal behavior model yet still carries out the same malicious action. Instead of using short sequences and being limited by length, state-based models use finite state machine (FSM) to express possible sequences, where the PC information (i.e., program counters which are the return addresses of system-calls) are often used in these models.

The design paradigm of Orpheus is to augment physical event constraints on top of an existing program behavior model. For example, automaton/state-based models can be enhanced with event checking on event-dependent state transitions. For the ngram model [32], we identify event-dependent n-grams in the training phase and apply the event checking when observing any event-dependent $\mathrm{n}$-gram in the monitoring phase. In addition, control-flow integrity [18], [35] can also be augmented with event checking before executing control flow transfers. We leave the option of choosing the underlying basic program behavior model open to system developers, which may depend on the specific resource constraints on CPS platforms. For example, compared with the n-gram model, tracing PC information in FSA/HMM during program execution incurs an extra runtime overhead, which we will demonstrate in Sec. 8. We instantiate the Orpheus framework using the FSA model [44] in Sec.5

\section{Reasoning About Cyber-Physical Execu- TION SEMANTICS}

In this section, we present a general method for reasoning about cyber-physical execution semantics of a control program through static analysis, including the event identification and dependence analysis.

\subsection{Event Identification}

In order to discover the triggering relationship between external events and internal program control flows, we first identify what events are involved in a control program. For pre-defined binary events, it is not difficult to identify these events (e.g., given event functions declared in an event library or header file, we scan the source code or executable binary). The main challenge is to identify: i) control intensity events/loops, and ii) non-predefined binary events. Our LLVM-based [47] event identification algorithm can automatically extract these events and only requires knowledge of sensor-reading APIs and actuation APIs on the embedded system. They are pre-specified sources and sink $\mathrm{S}^{3}$ in our static analysis.

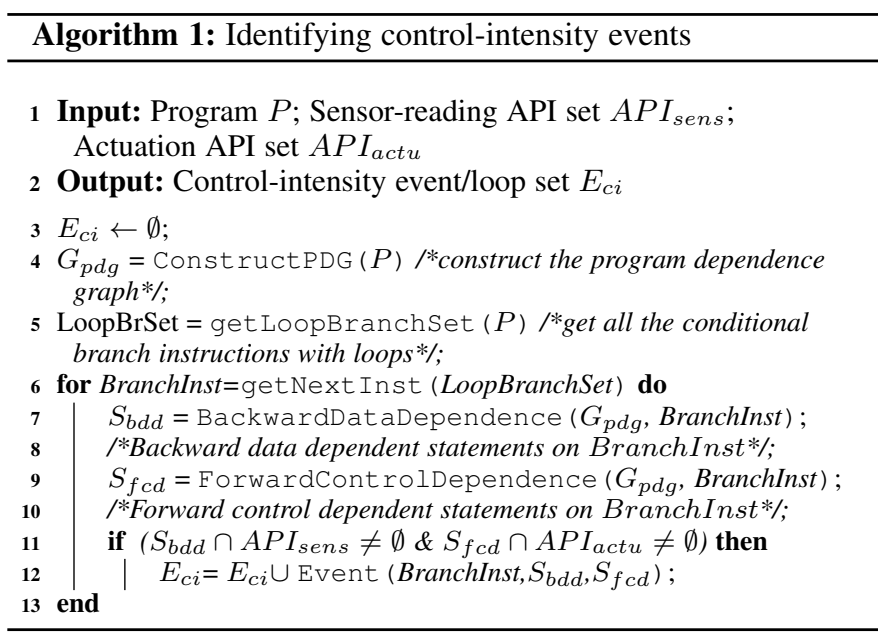

According to the definition of a control-intensity event in Sec. 2.1. it contains a loop statement (e.g., for/while loop) in which sensor values affect the amount of control operations. Our key idea is to search for a loop statement that is data-dependent on any sensor-reading API, and at least an actuation API is control-dependent on this loop statement. The search is performed through backward data dependence analysis and forward control dependence analysis. Algorithm 1 describes our static analysis for identifying control-intensity events. We first obtain the LLVM Intermediate Representation (IR) of a control program $P$ using the Clang compiler [47], and construct the program dependence graph (PDG), including both data and control dependencies (Line 4). The control dependence graph is at the basic block leve ${ }^{4}$ while the data dependency graph is at the granularity of instructions. Then, we obtain all conditional branch instructions with loops, by searching the conditional "br" instruction, which takes a single "i1" value and two "label" values in LLVM IR (Line 5). For each conditional branch with a loop, we conduct the backward interprocedural dataflow analysis to find any prior data dependence on sensor-reading APIs (Line 7). Then, we conduct the forward inter-procedural control-dependence analysis on the true branch of the conditional instruction to find actuation APIs, e.g., APIs in WiringPi library or functions writing GPIO pins [49] (Line 9). If a loop statement is data-dependent on external sensor data, and triggers a certain control action, we identify a control-intensity event/loop (Line 11). In each iteration, we record the identified control-intensity event and control intensity loop (Line 12), which is the output of the event identification process.

A more specific example of our event identification is illustrated in Fig. 3 corresponding to the $\mathrm{C}$-based control program in Listing 1. The figure shows a control-intensity event/loop represented by LLVM IR after the data dependence and control dependence analysis (1). We then locate a conditional branch instruction with a loop (2). This conditional branch uses the variable steps, which is data dependent on a sensor-reading API (3). On its true branch, we find an actuation API digitalWrite and thus we identify the loop as a control-intensity event (4).

3. Source and sink are terms in a dataflow/taint analysis. The source is where data comes from, and the sink is where it ends in a program [48].

4. In program analysis, a basic block is a linear sequence of instructions containing no branches except at the very end. 


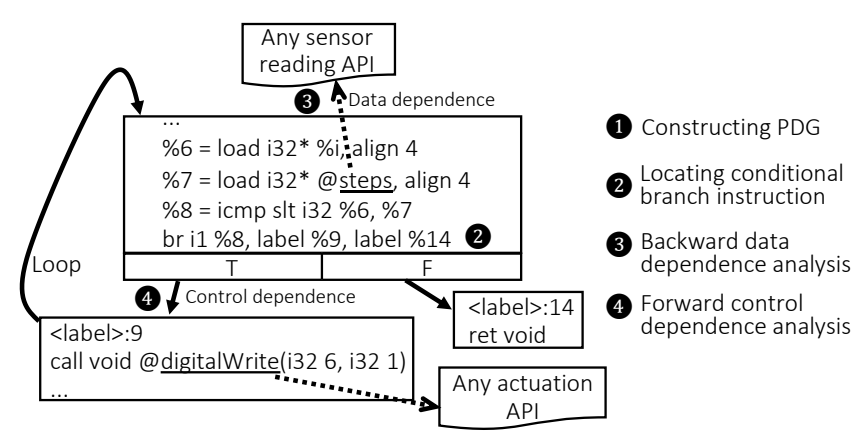

Fig. 3: An example of identifying control-intensity events

We also design a similar procedure for identifying non-predefined binary events. An example of such event is when the humidity exceeds a user-designated value, an event predicate returns True. In this procedure, we search for the conditional branch either "br" or "switch" instruction without a loop, and then perform the same data/control dependence analysis. In particular, we need to analyze both true and false branches of a "br" instruction, because both branches may contain control actions and we also consider the not-happening case (i.e., the branch without triggering any control action) as an implicit event.

\subsection{Event Dependence Analysis}

Our event dependence analysis generates an event-annotated CFG, i.e., approximating the set of statements/instructions that connect events and their triggered actions. During the event identification, we identify individual events that are involved in a control program. For the control-intensity event/loop, we directly associate it with the whole loop that contains the sensor-driven control action. A challenge arises when dealing with nested binary events. We address the nested events challenge using a bottom-up approach for recursive searching for event dependencies.

Algorithm 2 describes our event dependence analysis for nested binary events. Given a binary-event triggered basic block $B B_{\text {eta }}$, we backward traverse all its control dependent blocks until reaching the root in a recursive manner, and extract corresponding branch labels (i.e., True or False). In the recursive function FindEventDependence (Line 5), once we find a basic block on which $B B_{c u r}$ is control dependent (Line 7), we check whether it contains any external event (Line 9). If yes, we add this event together with its branch label to $E_{b}$ (Line 10). The condition $E_{b} \cap E_{t m p}=\emptyset$ avoids potential loops when including new events into $E_{b}$. Then, we recursively search any upstream event that $B B_{\text {cur }}$ depends on (Line 12).

Fig. 4 illustrates an example of our event dependence analysis corresponding to Listing 1 Block 7 (i.e., the basic block with label 7) is control dependent on Block 4 in the True branch of pull_event (called true-control-dependent). By backward traversing the control dependence graph, we find Block 4 is further false-control-dependent on push_event in Block 0 . Then, we know Block 7 is control dependent on a composite event [push_event $\wedge$ pull_event]. In this example, we also find Blocks 3 control dependent on push_event, and Block 9 is control dependent on $[\overline{\text { push_event }} \wedge \overline{\text { pull_event }}$. We finally identify three event-dependent basic blocks, and obtain the corresponding event-annotated CFG.

In addition to the static analysis approach, an alternative for event dependence analysis is using dynamic slicing [50], which
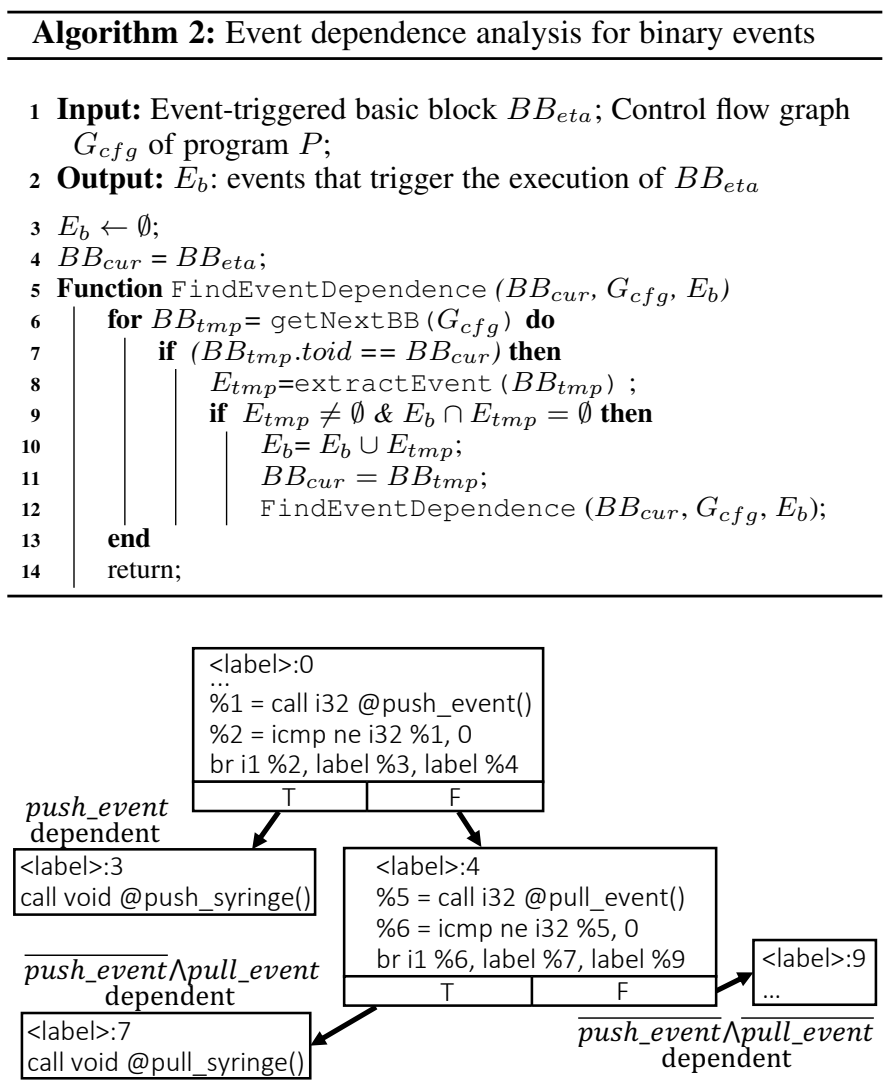

Fig. 4: Event dependence analysis for nested events

identifies statements triggered by a particular event during multiple rounds of program executions. It is worth mentioning that our event identification and dependence analysis is a general approach for reasoning cyber-physical execution semantics, independent of specific program anomaly detection models.

\section{5 eFSA: AN INSTANTIATION OF Orpheus}

In this section, we describe details about how to build the eventaware finite-state automaton (i.e., eFSA) model, a system call level FSA-based instantiation of the Orpheus framework. $e$ FSA captures the event-driven feature of CPS programs to detect evasive attacks.

\subsection{Formal Description of eFSA}

We construct the finite-state automaton (FSA) [44] model, which is based on tracing the system calls and program counters (PC) made by a control program under normal execution. Each distinct PC (i.e., the return address of a system call) value indicates a different state of the FSA, so that invocation of same system calls from different places can be differentiated. Each system call corresponds to a state transition. Since the constructed FSA uses memory address information (i.e., PC values) in modeling program behaviors (called the gray-box model), it is more resistant to mimicry attacks than other program models [16], [51].

In an execution trace, given the $k_{t h}$ system call $S_{k}$ and the PC value $p c_{k}$ from which $S_{k}$ was made, the invocation of $S_{k}$ results in a transition from the previous state $p c_{k-1}$ to $p c_{k}$ which is labeled with $S_{k-1}$. Fig. 5. a) shows a pictorial example program corresponding to Listing 1 at the system-call level, where system-calls are denoted by $S_{0}, \ldots, S_{6}$, and states are represented 
by integers (i.e., line numbers). Suppose we obtain three execution sequences, $\frac{S_{0}}{1} \frac{S_{1}}{3} \frac{S_{2}}{6} \frac{S_{3}}{7} \frac{S_{2}}{6} \frac{S_{3}}{7} \frac{S_{5}}{10} \frac{S_{6}}{11}, \frac{S_{0}}{1} \frac{S_{1}}{3} \frac{S_{4}}{9} \frac{S_{4}}{9} \frac{S_{5}}{10} \frac{S_{6}}{11}$, and $\frac{S_{0}}{1} \frac{S_{1}}{3} \frac{S_{5}}{10} \frac{S_{6}}{11} \frac{S_{1}}{3} \frac{S_{5}}{10} \frac{S_{6}}{11}$, the learnt FSA model is shown in Fig. 5(b), where each node represents a state and each arc represents a state transition.

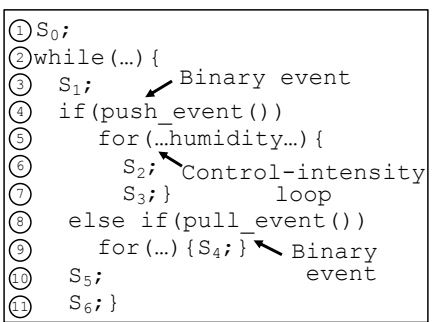

(a)

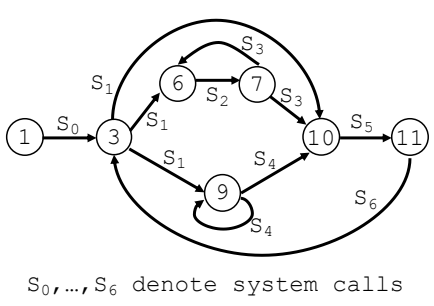

(b)
Fig. 5: System-call based finite-state automaton (FSA) model: (a) the example program of Listing 1 at the system-call level; (b) the corresponding FSA model.

Our $e$ FSA model extends FSA with external context constraints, where event-dependent state transitions in FSA are labeled with event constraints. We formally define the $e$ FSA model as a six-tuple: $\left(S, \Sigma, s_{0}, F, E, \delta\right) . S$ is a finite set of states which are PC values, and $\Sigma$ is a finite set of system calls (i.e., input alphabet). $s_{0}$ is an initial state, and $F$ is the set of final states. $E$ represents a finite set of external events, which can affect the underlying execution of a control program. $\delta$ denotes the transition function mapping $S \times \Sigma \times E$ to $S$. Note that a state transition may come with multiple physical events (referred to as a composite event). Thus, the input alphabet can be expressed as a cartesian product: $E=E_{1} \times E_{2} \times \cdots \times E_{n}$, where the input $E$ consists of $n$ concurrent physical events. In particular, we consider the nonoccurrence (not-happening) of one or more events as an implicit event in $e \mathrm{FSA}$.

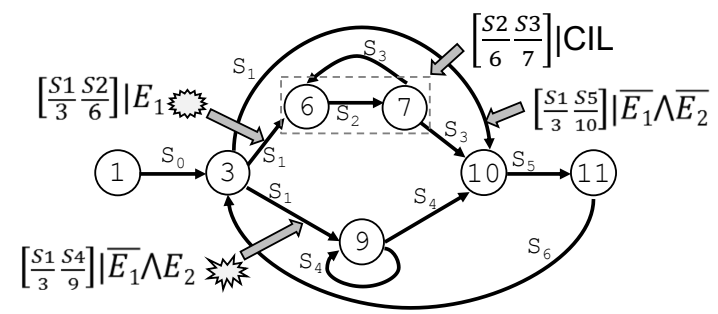

Fig. 6: An example of the $e$ FSA model, where $E_{1}$ represents push_event, $E_{2}$ represents pull_event, and CIL represents the control-intensity event/loop.

Fig. 6 shows an example of $e$ FSA model corresponding to the FSA example in Fig. 5, where an event dependent transition is labeled by "[ $\left.\frac{\text { System } C a t l}{P C}\right] \mid$ Events". In this example, there are two binary events and one control-intensity event. Through the event dependence analysis, we identify that lines 5-7 (where $S_{2}$ and $S_{3}$ are invoked) and line 9 (where $S_{4}$ is invoked) are dependent on the binary events $E_{1}$ and $E_{2}$, respectively. To avoid redundancy, we associate a binary event to the first state transition in FSA that is dependent on it. In Fig. 6 we identify binary-event dependent state transitions $\left[\frac{S_{1}}{3} \frac{S_{2}}{6}\right]\left|E_{1},\left[\frac{S_{1}}{3} \frac{S_{4}}{9}\right]\right| \overline{E_{1}} \wedge E_{2}$, and a control-intensityevent dependent control intensity loop $\left[\frac{S_{2}}{6} \frac{S_{3}}{7}\right] \mid C I L$. It also contains an implicit event dependent transition $\left[\frac{S_{1}}{3} \frac{S_{5}}{10}\right] \mid\left(\overline{E_{1}} \wedge \overline{E_{2}}\right)$.

\subsection{From Event-Annotated CFG to eFSA}

To construct an $e$ FSA model, we need to identify event-dependent state transitions at the system call level in FSA. Towards this end, we apply the event dependence analysis results (described in Sec. 4.1 and 4.2 to transform instruction-level dependencies in LLVM IR to the state transition dependencies in FSA. Such a mapping might be achieved through static analysis, e.g., passing over the parse tree to search for system call invocations. However, a static analysis based approach requires the modifications of gcc compiler or system call stubs, and even requires handcrafted modifications for library functions [52], [53]. In $e$ FSA, we adopt a dynamic profiling based approach to discover event dependent state transitions. We first transform instruction-level event dependencies in LLVM IR to statement-level dependencies in source code with line numbers. Then, we map line numbers and file names to return addresses (e.g., by using the addr2line tool) that are collected in the dynamic profiling phase when the FSA model is constructed. In this way, we obtain the system call level event-dependent state transitions in FSA. Subsequently, we augment the event-driven information over the underlying FSA, and finally construct the $e$ FSA model.

\subsection{Security Policies in eFSA}

$e$ FSA expresses causal dependencies between physical events and program control flows. By checking execution semantics (i.e., enforcing cyber-physical security policies) at runtime, eFSA improves the robustness against data-oriented attacks by increasing the difficulties that an attack could bypass the anomaly detection.

For state transitions that are dependent on binary events, the cyber-physical policy enforcement is to make sure the return values of binary events reflects the ground truth sensor measurements. For control intensity loops that are dependent on controlintensity events, our approach is based on the control intensity analysis, which models the relationship between the observable information in cyber space (i.e., system-calls) and sensor values in physical space. $e$ FSA then enforces the policy that the observed control intensity should be consistent with the trend of sensor value changes.

\subsection{Control Intensity Analysis}

The main challenge for detecting runtime control intensity anomalies lies in that, given system call traces of a control program, we need to map the control intensity to its reflected sensor measurements, where only the number of loop iterations in a control intensity loop is available. To this end, we first obtain the number of system calls invoked in each loop iteration. Then, we model the relationship between sensor measurements and the amount of system calls in a control intensity loop through a regression analysis.

Execution Window Partitioning and Loop Detection: Typically, control programs monitor and control physical processes in a continuous manner, where the top-level component of a program is composed of an infinite loop. For instance, an Arduino program [54] normally consists of two functions called setup () and loop(), allowing a program consecutively controls the Arduino board after setting up initial values. We define an execution window as one top-level loop iteration in a continuous program, and a behavior instance as the program activity within an execution window. The term execution window is equivalent to the scan cycle in industrial control domain [34]. We partition 
infinite execution traces into a set of behavior instances based on the execution window. The underlying FSA model helps identify loops since it inherently captures program loop structures. We first identify the starting state in the top-level loop of a FSA. Then, once a top-level loop back edge is detected, a behavior instance is obtained.

Regression Analysis: The purpose of the regression analysis is to quantify the relationship between sensor measurements and system call amount in a control intensity loop. Given the number of system calls invoked in each loop iteration, one straightforward approach is through manual code analysis. In this work, we present an approach for automating this process. During the identification of control-intensity events in Sec. 4.1. we know what sensor types (i.e., sensor reading APIs) are involved in a control intensity loop. In the training phase, we collect normal program traces together with the corresponding sensor values. Then, we perform a simple regression analysis to estimate the relationship between the system call amount (i.e., outcome) and sensor measurements (i.e., explanatory variables) for each control intensity loop. For example, suppose a control intensity loop is triggered by the change of humidity sensor value (details are in Sec. 8.4). We observe that an increase of humidity results in more iterations of the control intensity loop, where each loop iteration incurs 3 system calls. Thus, we can reversely derive the changes of physical environment by observing the number of iterations in a control intensity loop.

\section{EFSA-BASED DETECTION}

In this section, we present how an $e$ FSA-based anomaly detector detects anomalies particularly caused by data-oriented attacks, and discuss about the design choices of event verification.

\subsection{Runtime Monitoring and Detection}

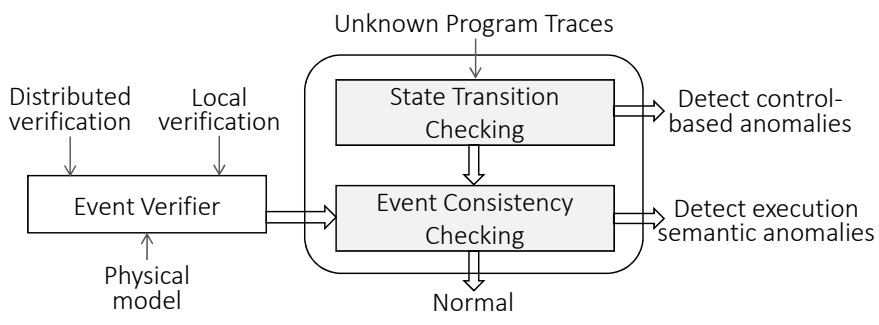

Fig. 7: $e$ FSA-based anomaly detection

Our anomaly detector traces system calls as well as the corresponding PC values during the execution of a control program. As shown in Fig. 7, the anomaly detection is composed of an event verifier and two checking steps: i) state transition integrity checking against the basic FSA model, and ii) event consistency checking against the event verification in the $e$ FSA-based anomaly detector, which is our new contribution.

- Event-independent state transition. For each intercepted system call, we check if there exists an outgoing edge labelled with the system call name from the current state in FSA. If not, an anomaly is detected. If the current state transition is not event-dependent, we move the current state of the automaton to the new state. This basic state-transition checking has been shown to be effective against common types of control-oriented attacks (e.g., code injection attacks or code-reuse attacks [15]) which violate control flow integrity of the model.
- Event-dependent state transition. In case of an event dependent state transition according to the $e$ FSA model, we first perform the above basic state-transition checking. More importantly, with the help of the event verification (discussed in Sec. 6.2), we then check the consistency between the runtime execution semantics and program's behavior, i.e., whether a specific physical event associated with this event-dependent state transition is observed in the physical domain. This step can detect stealthy data-oriented attacks that follow valid state transitions but are incompatible with the physical context. Another important aspect is the selection of event checkpoints. To avoid redundant checking, we set the checkpoint for a binary event at its first event-dependent state transition. For a controlintensity event, we perform the event checking after it jumps out of the control intensity loop.

\subsection{Event Verification Strategies}

The objective of event verification is to detect event spoofing caused by runtime data-oriented software exploits. Event verification is highly application specific, and it is actually orthogonal to the $e$ FSA model itself. We describe several possible approaches for verifying physical context.

- Local event verification: which is able to detect the inconsistency between program runtime behavior and cyber-physical execution semantics. For example, the monitor re-executes a binary-event function to confirm the occurrence of the event. To detect control intensity anomalies, the monitor retrieves sensor measurements and compares them against the derived sensor values from system call traces. There may exist false positives/negatives due to sensor's functional failures in practice.

- Distributed event verification: which assesses the physical context by exploiting functionally and spatially redundancy of sensors among co-located embedded devices. Since sensor data normally exhibit spatio-temporal correlation in physical environments, it increases the detection accuracy by involving more event verification sources.

- Physical model based verification: which is complementary to the runtime event verification. Cyber-physical inconsistency may be detected based on physical models [55]. For example, one may utilize fluid dynamics and electromagnetics as the basic laws to create prediction models for water system [56] and power grid [57]. Based on the prediction models and predefined threat constraints, these methods can then check whether the predicted environment values are consistent with a control system's behavior.

\section{IMPLEMENTATION}

To demonstrate the feasibility of our approach, we have implemented a prototype with around $5 \mathrm{~K}$ lines in $\mathrm{C} / \mathrm{C}++$, Bash and Python codes, including the trace collection and preprocessing, event identification and dependence analysis, $e$ FSA model construction, and runtime anomaly detection modules. Our prototype uses multiple off-the-shelf tools and libraries in Linux.

We choose Raspberry Pi 2 with Sense HAT as the main experimental platform, which is a commonly used platform for building embedded control applications [7], [34], [35]. Sense Hat, an add-on board for Raspberry Pi, provides a set of environmental sensors to detect physical events including pressure, temperature, humidity, acceleration, gyroscope, and magnetic filed. During the 
training phase, we collect program traces on Raspberry Pi and perform the $e$ FSA model construction on a Linux Desktop (Ubuntu 16.04, Intel Xeon processor $3.50 \mathrm{GHz}$ and $16 \mathrm{~GB}$ of RAM). In the monitoring phase, the anomaly detector is deployed on Raspberry Pi to detect runtime control-based or data-oriented attacks. In the following, we present key implementation aspects in our prototype.

Dynamic Tracing. We use the system tool strace-4.13 to intercept system call of a running control program. To obtain the $\mathrm{PC}$ value from which a system call was invoked in a program, we need to go back through the call stacks until finding a valid PC along with the corresponding system call. We compile strace with - libunwind support, which enables stack unwinding and allows us to print call stacks on every system call.

Event Identification and Dependence Analysis. Our event identification and dependence analysis tool is implemented within the Low Level Virtual Machine (LLVM) ${ }^{5}$ compiler infrastructure, based on an open source static slicer ${ }^{6}$ which builds dependence graph for LLVM bytecode. An advantage of using LLVM-based event dependence analysis is that, our tool is compatible with multiple programming languages since LLVM supports a wide range of languages. Our event identification module identifies the line numbers in source code where an event is involved. Then, the event dependence analysis outputs the line numbers of event dependent statements.

Anomaly Detector with Event Verification. In our prototype, we implement a proof-of-concept near-real-time anomaly detector using named pipes on Raspberry $\mathrm{Pi}$, including both local and distributed verifications (corroboration with single or multiple external sources). We develop a sensor event library for Raspberry Pi Sense Hat in $\mathrm{C}$ code, based on the sensor reading modules in experix $7^{7}$ and $c$-sense-hat ${ }^{8}$. The event library reads pressure and temperature from the LPS25H sensor, and reads relative humidity and temperature from the HTS221 sensor, with maximum sampling rates at 25 per second. Our local event verifier calls the same event functions as in the monitored program, and locally check the consistency of event occurrence. In the distributed event verifier, we deploy three Raspberry Pi devices in an indoor laboratory environment. We develop a remote sensor reading module which enables one device to request realtime sensor data from neighbouring devices via the sockets communication.

\section{EXPERIMENTAL VALIDATION}

We conduct CPS case studies, and evaluate eFSA's detection capability against runtime data-oriented attacks. Our experiments aim to answer the following questions:

- What is the runtime performance overhead of $e \mathrm{FSA}$, including the model training overhead, system-call tracing overhead and detection latency (Sec. 8.2)?

- Whether $e$ FSA is able to detect different data-oriented attacks (Sec. 8.3 and 8.4 ?

- How feasible is the event-aware n-gram model as an alternative instantiation of the Orpheus framework (Sec. 8.5)? Whether $e \mathrm{FSA}$ can be generalized to detect network event injection attacks (Sec. 8.6)?

5. http://llvm.org/

6. https://github.com/mchalupa/dg

7. http://experix.sourceforge.net/

8. https://github.com/davebm1/c-sense-hat

\subsection{CPS Case Studies}

Solard 9 . It is an open source controller for boiler and house heating system that runs on embedded devices. The controller collects data from temperature sensors, and acts on it by controlling relays via GPIO (general purpose input/output) pins on Raspberry Pi. Control decisions are made when to turn on or off of heaters by periodically detecting sensor events. For example, CriticalTempsFound() is a pre-defined binary event in Solard. When the temperature is higher than a specified threshold, the event function returns True.

SyringePump ${ }^{10}$. It was developed as an embedded application for Arduino platform. Abera et al. [7] ported it to Raspberry Pi. The control program originally takes remote user commands via serial connection, and translates the input values into control signals to the actuator. SyringePump is vulnerable since it accepts and buffers external inputs that might result in buffer overflows [7]. We modify the syringe pump application, where external inputs are sent from the control center for remote control, and environmental events drive the pump's movement. Specifically, in the event that the relative humidity value is higher than a specified threshold, the syringe pump movement is triggered. In addition, the amount of liquid to be dispensed is linearly proportional to the humidity value subtracted by the threshold. Such sensor-driven syringe pumps are used in many chemical and biological experiments such as liquid absorption measurement experiment.

\subsection{Training and Runtime Performance}

In the training phase, we collect execution traces of Solard and SyringePump using training scripts that attempt to simulate possible sensor inputs of the control programs. By checking Solard and SyringePump's source codes, our training scripts cover all execution paths.

We first measure the time taken for training models in our prototype, where the main overhead comes from the event dependence analysis. Table 1 illustrates $e$ FSA's program analysis overhead in the training phase. For comparison purpose, we deploy the LLVM toolchain and our event dependence analysis tool on both Raspberry Pi and Desktop Computer (Intel Xeon processor $3.50 \mathrm{GHz}$ and 16GB of RAM). From Table 1 . Raspberry Pi takes much longer time (more than 150 times) than desktop computer to complete the program dependence analysis task. It only takes $0.745 \mathrm{~s}$ and $0.0035 \mathrm{~s}$ for event dependence analysis of Solard (46.3 $\mathrm{kb}$ binary size) and SyringePump (17.7 kb binary size) on a desktop computer, respectively. Since Solard and SyringePump run in a continuous manner and thus generate infinite raw traces. The model training overhead is measured by how much time it takes for training per MByte raw trace. Results show that it takes less than $0.2 \mathrm{~s}$ to process 1 MByte traces on the desktop computer. The number of states in Solard's and SyringePump's $e$ FSA is 34 and 65, respectively (not including system-calls in the initialization before entering the main function).

\begin{tabular}{ccc}
\hline & \multicolumn{2}{c}{ Event Dependence Analysis } \\
\cline { 2 - 3 } & Desktop Computer & Raspberry Pi 2 \\
\hline \hline Solard & $0.745 \mathrm{~s}$ & $109.975 \mathrm{~s}$ \\
\hline SyringePump & $0.0035 \mathrm{~s}$ & $1.726 \mathrm{~s}$ \\
\hline
\end{tabular}

TABLE 1. Average delay overhead in training phase

9. https://github.com/mrpetrov/solarmanpi

10. https://github.com/control-flow-attestation/c-flat 
Next, we measure the performance overhead incurred by $e$ FSA's anomaly detector on Raspberry $\mathrm{Pi}$, including the systemcall tracing overhead and anomaly detection overhead. The system-call tracing overhead has no difference between FSA and $e$ FSA, which incurs $1.5 \mathrm{x} \sim 2 \mathrm{x}$ overhead in our case studies. To comprehensively measure the runtime system-call tracing overhead, we further experimentally compare the tracing overhead on Raspberry Pi using three utility applications (i.e., tcas (1608 test cases), replace (5472 test cases), and schedule (2650 test cases)) from the Software-artifact Infrastructure Repository (SIR) benchmark suite [58]. Fig. 8 shows the results, which measure the elapsed time between the entry and exit points in the three utility applications. The baseline refers to the execution time without tracing. The runtime performance overhead of strace shows around $96 \%$ slowdown on average. When tracing the callstack information on every system-call, it yields around $112 \%$ slowdown. We discuss the tracing overhead limitation in Sec. 9

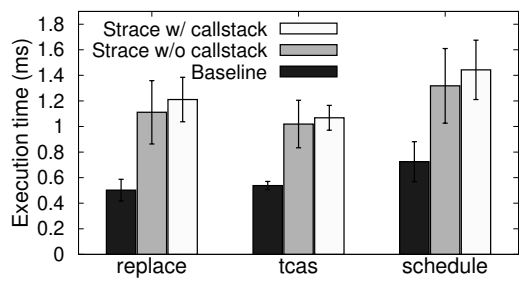

Fig. 8: System-call tracing overhead on Raspberry Pi

Table 2 reports the runtime anomaly detection latency results. The average delay for each state transition (i.e., each intercepted system call) checking out of more than 1000 runs is around $0.0001 \mathrm{~s}$. It takes $0.063 \mathrm{~s}$ on average to perform the local event checking. The end-to-end latency for the distributed event checking from each co-located device can be broken down into two main parts: i) network communication around $0.042 \mathrm{~s}$, and ii) sensor reading delay around $0.0582 \mathrm{~s}$. In our experiment, we deploy two co-located devices, and thus the total distributed event checking delay is around $0.212 \mathrm{~s}$. It is expected that the overhead of distributed event checking is linearly proportional to the number of event verification sources.

\begin{tabular}{ccc}
\hline Delay (Raspberry Pi 2) & Mean & Standard Deviation \\
\hline \hline FSA State Transition Checking & $0.00013293 \mathrm{~s}$ & $0.00004684 \mathrm{~s}$ \\
\hline Local Event Verification & $0.06279120 \mathrm{~s}$ & $0.00236999 \mathrm{~s}$ \\
\hline Distributed Event Verification & $0.21152867 \mathrm{~s}$ & $0.03828739 \mathrm{~s}$ \\
\hline
\end{tabular}

TABLE 2. Runtime detection overhead in the monitoring phase

\subsection{Detecting Attacks on Control Branch}

In this experiment, we evaluate $e$ FSA's security guarantees against control branch attacks.

\subsubsection{Solard}

In Solard, we engineer a buffer overflow vulnerability and manipulate the temperature sensor values to maliciously prevent the heater from being turned off. This cyber-physical attack is similar to the recent real-world German steel mill attack [28], which may result in a blast furnace explosion. In this experiment, we attach the Raspberry $\mathrm{Pi}$ on an electric kettle (i.e., 1-Liter water boiler). The control program keeps monitoring temperature values. When the temperature is lower than $50^{\circ} \mathrm{C}$, it turns on the heater. And when the temperature is higher than $60^{\circ} \mathrm{C}$, where CriticalTempsFound () is supposed to return True, it turns

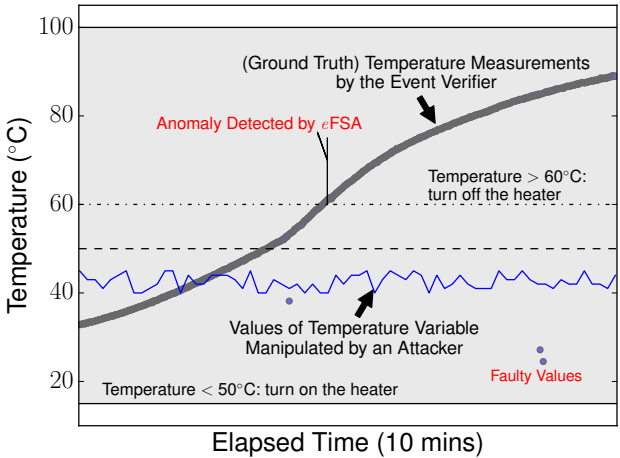

Fig. 9: An instance of Solard experiment

off the heater. In the monitoring phase, when we detect an eventdependent state transition in $e$ FSA model, the local event verifier performs event consistency checking.

Fig. 9 illustrates an instance of the Solard experiment. We corrupt the temperature sensor values in the range of $40 \sim 45^{\circ} \mathrm{C}$, which falsifies the return value of CriticalTempsFound () to be always False. In every scan cycle, $e$ FSA observes a state transition dependent on the not-happening of CriticaltempsFound() (i.e., an implicit event), and thus the event verifier checks the instantaneous temperature value. In our experiment, because the Raspberry Pi does not physically interact with the electric kettle, the ground truth temperature keeps increasing up to more than $80^{\circ} \mathrm{C}$ in Fig. 9 However, $e$ FSA successfully raises an alarm at the first moment when it finds a mismatch between the execution semantics (temperature exceeding $60^{\circ} \mathrm{C}$ ) and program behavior.

We did encounter sensor measurement failures, e.g., isolated dots as shown in Fig. 9 On average, the false sensor measurement rate is lower than $1 \%$ in our experiments. This means that the detection rate and false positive/negative rate would depend on sensors' functional reliability in practice. Existing methods, such as data fusion [59] can be applied to enhance the detection accuracy.

\subsubsection{SyringePump}

In SyringePump, we set the threshold to $40 \mathrm{rH}$, i.e., when the relative humidity value is higher than $40 \mathrm{r} \mathrm{H}$, it drives the movement of syringe pump by sending control signals to dispense liquid. The buffer overflow attack manipulates the humidity sensor values to purposely trigger event-push control actions without receiving an external event or environmental trigger. Such an attack leads to unintended but valid control flows.

Fig. 10 illustrates an example of the experiment. The remote user command corrupts the humidity sensor value to be $48.56 \mathrm{rH}$, which falsifies the return value of event-push to be True. In case of any event-driven state transition according to $e \mathrm{FSA}$, the event verifier checks consistency between the runtime execution semantics (e.g., the instantaneous humidity value) and program internal state. As shown in Fig. 9 eFSA raises an alarm when it finds a mismatch between the execution semantics and program behavior.

\subsection{Detecting Attacks on Control Intensity}

In this experiment, we demonstrate that $e \mathrm{FSA}$ is able to detect control intensity attacks with only system call traces. In SyringePump, we set the threshold that triggers the movement of syringe pump 


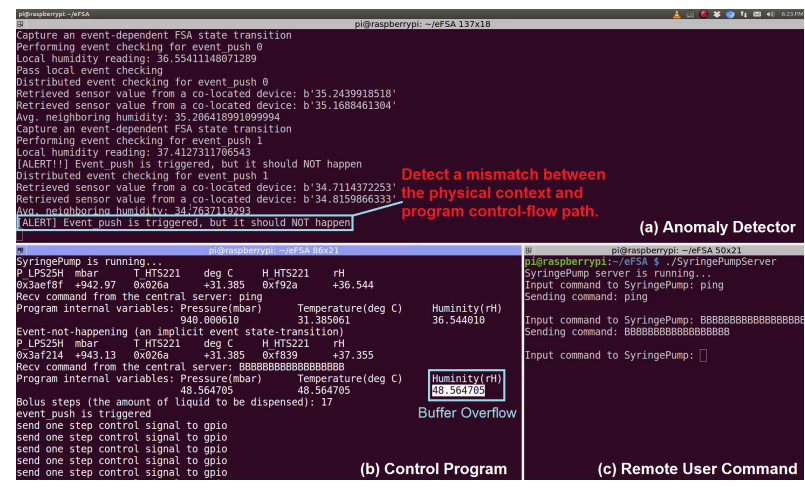

Fig. 10: An instance of SyringePump experiment

to be $30 \mathrm{rH}$. The corrupted humidity value determines the amount of liquid to be dispensed, which equals to the humidity value subtracted by $30 r H$ in this test. In the training stage, we obtain the number of system calls invoked in each loop iteration. Then, we model the relationship between sensor measurements and the amount of system calls in a control intensity loop. Through control intensity analysis, we know the number of system calls with no event occurrence is 40 per scan cycle, and each loop iteration (i.e., dispensing a unit of liquid) in the control intensity loop corresponds to 3 system-calls write-nanosleep-nanosleep, as shown in Fig. 11

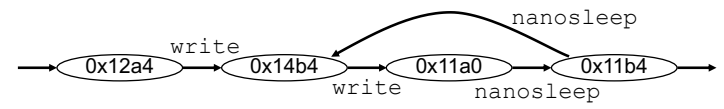

Fig. 11: Control intensity loop in $e$ FSA of SyringePump

Fig. 12 a) shows the value changes of the humidity variable and system call amount per scan cycle of SyringePump. The normal humidity value fluctuates between $34 \mathrm{rH}$ and $38 \mathrm{rH}$. As a result, the amount of liquid to be dispensed is subsequently changed, which is reflected by the number of system calls in each control loop. We manipulate the humidity values to be $20 \mathrm{rH}$ and $48 r H$, respectively. In the monitoring phase, by observing the number of system calls in each control loop, we can reversely derive the changes of physical environment based on our control intensity regression model as shown in Fig. 12 b). In this test, if the difference between the derived value and the sampled average value from event verifier is larger than $3 r H$, we consider it an anomaly. By checking the humidity measurements from two colocated devices (i.e., denoted as devices 1 and 2), our distributed event verifier detects that the program's runtime behaviors are incompatible with physical contexts. Thus, eFSA successfully detects the control intensity attacks.

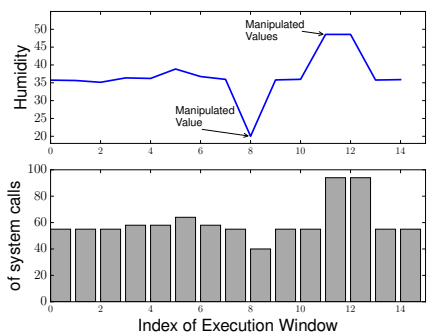

(a) Humidity and system call traces

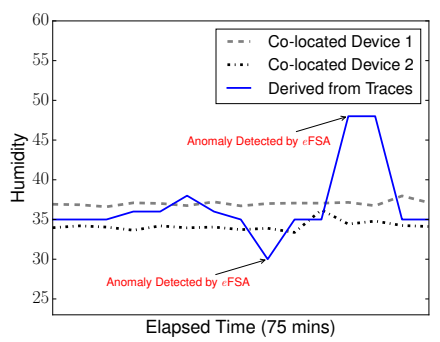

(b) $e$ FSA's detection

Fig. 12: An instance of SyringePump experiment with a sampling rate of 5 minutes

\subsection{Event-Aware N-gram Model}

To show the feasibility of augmenting the n-gram model with event awareness, we conduct the case study of the event-aware n-gram model, which is an alternative instantiation of the Orpheus framework. Given the execution traces of SyringePump in the training phase, we construct the n-gram model of system-call sequences $(\mathrm{n} \in[2, \cdots, 10])$. For the 2-gram model, there are 35 different 2grams. write-write is an event-dependent 2-gram. However, 15 non-event-dependent write-write system-call sequences are also observed during the monitoring phase, which makes the event checking no longer effective due to the ambiguity. This is because, without the PC (return address) information associated with each system-call, we cannot differentiate the invocation of the same system-calls from different places in the program. As a result, we need to increase the length of the n-gram until the event-dependent n-gram is unique in the model. But there is no guarantee we can always find the unique event-dependent $n$ gram with increasing the value of $n$. In SyringePump, we find a unique event-dependent 4-gram write-write-nanosleepnanosleep, where the model contains 60 different 4-grams. During the monitoring phase, our anomaly detector performs the event checking whenever this event-dependent 4-gram is observed. Nevertheless, with the increase of $n$, the size of the n-gram model also increases. For example, when we increase $n$ to 10 , the size of the n-gram model is increased to 96 , which is larger than the size of $e$ FSA. From this case study, we demonstrate that it is desirable to use the program counter (PC) information in the basic program behavior model, which significantly increases the resistance against control-flow attacks, but also resolves ambiguities in our event-aware anomaly detection.

\subsection{Extension of eFSA}

Control programs running on embedded devices may receive network events from the control center, and then execute actuation tasks. Though $e$ FSA mainly detects software-exploit based physical event spoofing, it is also applicable to network event-triggering scenarios. For example, a ground control station (GCS) sends a command to control an unmanned aerial vehicle (UAV) to change its flight mode. In this case, we consider each type of control command as a specific event, and the $e \mathrm{FSA}$ model of a UAV program is augmented with both physical sensing and network command events. Such an $e$ FSA model can detect false command injection attacks against the UAV by checking the consistency of system call traces at a UAV and its GCS, ensuring their system call invocations conforming to the network API semantics [60].

To demonstrate the applicability of $e$ FSA for detecting network event inconsistency in CPS, we conduct an experiment on AR.Drone ${ }^{11}$ which is a remote controlled quadrocopter. Different from the Solard and SyringePump case studies running on Raspberry Pi, AR.Drone runs on a customized mainboard with embedded Linux. AR.Drone allows a connection to the Telnet port which leads to a root shell, and thus we are able to deploy the strace tool to collect system call traces of the UAV control program. In AR.Drone, the system call types involved in the process that handles remote commands are quite limited and the program logic is rather simple. Thus, we can easily construct the corresponding $e$ FSA model by taking advantage of network protocol interactions

11. https://www.parrot.com/us/drones/parrot-ardrone-20-elite-edition 
without program dependency analysis. Sample traces are included in the accompanying materials for this pape ${ }^{12}$.

Since AR.Drone runs with an open Wi-Fi, and we know the communication protocol between UAV and the GCS [61], we can easily launch a false command injection attack, which could be used to send malicious operational data such as status or control command. We use a laptop (as an attacker) to send fake control commands to the AR.Drone. Meanwhile, we monitor the network traffic at GCS using the Wireshark tool. The event verifier could find inconsistencies between $e$ FSA's state transitions and network events captured at GCS, and thus detect this type of attacks. In this experiment, we do not intend to use $e \mathrm{FSA}$ to raise an alarm at the time of intrusion, instead we aim at detecting the anomalous behavior as a post-mortem analysis.

\section{LIMITATION}

Although our work is focused on providing new security capabilities in control-program anomaly detection against data-oriented attacks, in this section, we examine the limitations of our implementation and discuss how our method can be deployed in the near future.

Bare-metal CPS Devices: Our anomaly detection system works on the granularity of system calls and it leverages dynamic tracing facilities such as the strace tool, which requires the operating system support. An important reason behind our choice is that, the new generation of embedded control devices on the market are increasingly coming with operating systems [35], [37]. For example, Raspberry Pi devices with embedded Linux OS have been used as field devices in many CPS/IoT applications [62]. Linux-based PLCs for industrial control have emerged to replace traditional PLCs [63] for deterministic logic execution. However, embedded devices may still operate in bare-metal mode [7], where we can not utilize existing tracing facilities to collect system call traces. For traditional PLCs, our security checking can be added to the program logic. We can also apply the event checking idea to an anomaly detection system at the level of instructions. We may instrument the original control program with event checking hooks by rewriting its binary, e.g., inserting hooks at the entry of event-triggered basic blocks. We consider it as the future work to extend our design paradigm for fine-grained anomaly detection with binary instrumentation.

Tracing Overhead and Time Constraints: Though system call traces are a common type of audit data in anomaly detection systems, we would like to point out that the conventional softwarelevel system call tracing incurs unnegligible performance overhead to the monitored process [64]. It holds for time-insensitive embedded control applications, e.g., smart home automation, but would be a technical challenge for time-sensitive applications. While we employ the user-space strace software to collect system calls in our prototype, tracing tools are orthogonal to our detection design. For performance consideration, alternative tracing techniques may be adopted in replacing strace to improve the tracing performance [39]. For example, it is possible to improve the performance for system call interposition by modifying the kernel at the cost of increased deployment effort. With the recently unveiled Intel's Processor Trace (PT) and ARM's CoreSight techniques, hardware tracing infrastructures are increasingly embedded in modern processors, which can achieve less than $5 \%$ performance

12. Sample system call traces are provided at goo.gl/Wkrdzz overhead [65]. The recent work, Ninja [66], offers a fast hardwareassisted tracing on ARM platforms. The overhead of instruction tracing and system call tracing are negligibly small. Therefore, we anticipate that future tracing overhead will be significantly reduced as the hardware-assisted tracing techniques are increasingly used.

Lack of CPS Benchmark Programs: Lack of CPS benchmark programs is one of the challenges in CPS security research to perform sound evaluation. It is partly because of the diversity of CPS hardware platforms, and the hardware-dependent nature of CPS programs. In addition, safety-critical CPS programs are rarely open-source. As a result, existing CPS security research work mainly conducted limited case studies [7] [43] [67], instead of a large scale experimental evaluation. We therefore leave the comprehensive evaluation of our approach for future work as more CPS benchmark programs are available. We also would like to exploit the symbolic execution in our control intensity analysis, which may statically derive the relationship between sensor values and the number of control loop iterations. Symbolic execution and fuzzing techniques are also useful to increase code-coverage in the training phase for collecting traces of normal program executions.

Limitation of Detection Capability: From our case studies, we demonstrate that enforcing cyber-physical execution semantics in CPS program anomaly detection is effective to detect both types of data-oriented attacks. The necessary condition is that the observed program behavior at system-call level is incompatible with the current physical context. Simply corrupting non-control data in a program's memory space may be undetectable if the attack does not result in inconsistencies between the physical context and program execution, which is out of the scope in this work. Another limitation of our design is that we only detect program anomalies at the system-call level. CPS devices may send control signals by directly writing registers without issuing any system-call, rendering the system-call based detection not working. To overcome this limitation, the Orpheus design paradigm can be extended to the instruction level for fine-grained anomaly detection.

Anomaly Detection as a Service: Embedded devices are resource-constrained compared with general-purpose computers. To reduce detection overhead, the anomaly detection may be performed at a remote server. We envision deployment involving partnerships between hardware vendors and security service providers (similar to ZingBox IoT Guardian [68]), where the security provider is given access to embedded platforms and helps clients to diagnose/confirm violations. The client-server architecture resonates with the remote attestation in embedded systems, which detects whether a controller is behaving as expected [7], [69]. For detection overhead reduction, the remote server may choose when and how frequently to send assessment requests to a control program for anomaly detection. It is also possible to selectively verify a subset of events for the scalability purpose, e.g., only safety-critical events specified by developers are involved. While the event verifier implementation is not completely automated, our event identification and dependence analysis tool does automate a large portion of event code extraction and eases the developer's burden. We leave automatically generating event verification functions for the anomaly detector as an important part of our future work.

\section{Related Work}

Due to the diversity of CPS applications, existing anomaly detection solutions are proposed to detect specific attacks for 


\begin{tabular}{|c|c|c|c|c|}
\hline Research Work & Category & Approach & Security Guarantee & Validation \\
\hline Yoon et al. 31] & $\begin{array}{c}\text { Program behavior model } \\
\text { (cyber) }\end{array}$ & Syscall frequencies & Frequency-based program control flow anomaly & Raspberry Pi testbed \\
\hline Feng et al. 74] & $\begin{array}{l}\text { Network traffic analysis } \\
\text { (cyber) }\end{array}$ & Machine learning based traffic analysis & Traffic alteration & $\begin{array}{l}\text { Traffic data from a gas pipeline } \\
\text { system }\end{array}$ \\
\hline Zimmer et al. [10] & $\begin{array}{l}\text { Timing analysis model } \\
\text { (cyber) }\end{array}$ & Static/dynamic timing analysis & Code injection attacks & Simulation/Testbed \\
\hline C-FLAT 7] & $\begin{array}{c}\text { Program behavior model } \\
\text { (cyber) }\end{array}$ & Program analysis and instrumentation & $\begin{array}{l}\text { Control-oriented attacks and limited } \\
\text { non-control-data attacks }\end{array}$ & Raspberry Pi testbed \\
\hline FT-RMT 67] & $\begin{array}{l}\text { Redundant execution } \\
\text { analysis (cyber) }\end{array}$ & Redundant controller and computation & CPS faults or attacks & $\begin{array}{c}\text { Simulate steer-by-wire application } \\
\text { on embedded Linux }\end{array}$ \\
\hline Hadziosmanovic et al. 56] & $\begin{array}{l}\text { Range-based model } \\
\text { (physical) }\end{array}$ & $\begin{array}{l}\text { Attribute values extracted from } \\
\text { network traffic }\end{array}$ & False data injection attacks & $\begin{array}{l}\text { Traffic data from water treatment } \\
\text { plants }\end{array}$ \\
\hline NoisePrint 75] & $\begin{array}{c}\text { Noise fingerprinting } \\
\text { (physical) }\end{array}$ & $\begin{array}{l}\text { Pattern recognition in sensor and } \\
\text { process noise dynamics }\end{array}$ & Sensor spoofing attacks & Two real-world CPS testbeds \\
\hline Cardenas et al. 4] & Physical laws & $\begin{array}{c}\text { Linear model derived from training } \\
\text { data }\end{array}$ & False data injection attacks & Simulation \\
\hline SRID 25] & Physical laws & $\begin{array}{l}\text { Correlation analysis of system } \\
\text { variables. }\end{array}$ & False data injection attacks & Simulation \\
\hline $\mathrm{C}^{2} 76$ & $\begin{array}{l}\text { Control policies } \\
\text { (physical) }\end{array}$ & User specified control policies & Control signal violation & Raspberry Pi testbed \\
\hline$e$ FSA (Our work) & Cyber-physical model & Event-aware FSA & Data-oriented attacks & Raspberry Pi testbed \\
\hline
\end{tabular}

TABLE 3. Comparison of representative CPS anomaly detection approaches

specific applications, such as smart infrastructures [3], unmanned aerial vehicles [70], medical devices [71], automotive [72], [73], industrial control process [4], [34], [55]. The majority of research efforts in this area thus far have concentrated on behavior modelbased anomaly detection [55], and can be generally classified into two categories: 1) cyber model (e.g., program behavior model, network traffic analysis, or timing analysis); 2) physical model (e.g., range-based model or physical laws). Our proposed $e \mathrm{FSA}$ analyzes both the cyber and physical properties of CPS, as well as their interactions. Thus, we refer to it as the cyber-physical model. Table 3 compares representative CPS anomaly detection solutions.

- Program behavior model. Regarding the CPS anomaly detection based on program behavior models in the cyber domain, Yoon $e t$ al. [31] proposed a lightweight method for detecting anomalous executions using the distribution of system call frequencies. The frequencies are for individual system calls, i.e., 1-grams. The authors in [20] proposed a hardware based approach for control-flow graph (CFG) validation in runtime embedded systems. McLaughlin et al. [34] presented the Trusted Safety Verifier (TSV) to verify safety-critical code executed on programmable controllers, such as checking safety properties like range violations and interlocks of PLC programs. C-FLAT [7] instruments target control programs to achieve the remote attestation of execution paths of monitored programs, and the validity of control flow paths is based on static analysis. Given an aggregated authenticator (i.e., fingerprint) of the program's control flow computed by the prover, the verifier is able to trace the exact execution path and thus can determine whether application's control flow has been compromised. C-FLAT [7] is the most related work to our approach. Both C-FLAT and $e$ FSA target at designing a general approach for detecting anomalous executions of embedded systems software. However, C-FLAT is insufficient to detect data-oriented attacks due to the lack of runtime execution context checking. It can only partially detect control intensity attacks with the assumption of knowing legal measurements of the target program. However, if the legal measurement covers a large range of sensor values, attacks can easily evade its detection because it does not check runtime consistency between program behavior and physical context.

- Traffic-based model. Control systems exhibit relatively simpler network dynamics compared with traditional IT systems, e.g., fixed network topology, regular communication patterns, and a limited number of communication protocols. As a result, implementing network-based anomaly detection systems would be easier than traditional mechanisms. Feng et al. [74] presented an anomaly detection method for ICS by taking advantage of the predictable and regular nature of communication patterns that exist between field devices in ICS networks. In the training phase, a base-line signature database for general packages is constructed. In the monitoring phase, the authors utilize Long Short-Term Memory (LSTM) network based softmax classifier to predict the most likely package signatures that are likely to occur given previously seen package traffic. The anomaly detector captures traffic anomalies if a package's signature is not within the predicted top $k$ most probable signatures according to the LSTM-based model.

- Timing-based model. Several studies utilized timing information as a side channel to detect malicious intrusions. The rationale is that execution timing information is considered an important constraint for real-time CPS applications, and mimicking timing is more difficult than mimicking the execution sequence. To this end, Zimmer et al. [10] used the worst-case execution time (WCET) obtained through static analysis to detect code injection attacks in CPS. Such timing-based detection technique is realized by instrumenting checkpoints within real-time applications. Sibin et al. [77] focused on detecting intrusions in realtime control systems. Yoon et al. [78| presented SecureCore, a multicore architecture using the timing distribution property of each code block to detect malicious activities in real-time embedded system. Lu et al. [21] investigated how to reduce timing checkpoints without sacrificing detection accuracy in embedded systems.

- Redundant execution analysis. Munir et al. [67] presented an integrated approach for the design of secure and dependable automotive CPS by conducting the steer-by-wire (SBW) case study. To provide fault tolerance (FT) to SBW applications, they proposed the FT-RMT (redundant multi-threading) scheme, which executes safety-critical computations on redundant threads and detects an error if observing a mismatch between the two threads' output. However, introducing redundant controllers into CPS incurs high cost such as increased code size and reduced performance. In addition, under our threat model, attackers may be able to compromise redundant controllers to evade detection.

- Range based model. Enforcing data ranges is the simplest method to detect CPS anomalies in the physical domain. As long as sensor readings are outside a pre-specified normal range, the anomaly detector raises an alarm. Hadziosmanovic et al. [56] presented a non-obtrusive security monitoring system by deriving models for PLC variables from network packets as the basis for assessing CPS behaviors. For constant and attribute 
series, the proposed detection approach raises an alert if a value reaches outside of the enumeration set. However, range-based detection suffers from a low detection rate because it neglects the program's execution context, e.g., if the legal measurement covers a large range of sensor values, attacks can easily evade its detection.

- Sensor and process noise fingerprinting. Ahmed et al. [75] proposed the NoisePrint, a CPS attack detection method based on process noise patterns (e.g., fluid sloshing in a storage tank) of the system. The intuition behind NoisePrint is that, sensor and process noise variations exhibit unique patterns among different processes. Therefore, it is hard for attackers to reproduce or control these noise variations, making them an ideal side information for CPS anomaly detection. In the training phase, a combined fingerprint dictionary for sensor and process noise is built under regular operations. Under sensor spoofing attack, noise pattern deviates from the fingerprinted pattern is considered an anomaly. However, this approach requires an accurate noise measurement, which is subject to the ambient background noise in a process plant.

- Physical laws. The idea of using physical models to define normal operations for anomaly detection is that, system states must follow immutable laws of physics. Wang et al. [25] derived a graph model to defeat false data injection attacks in SCADA system. It captures internal relations among system variables and physical states. Cho et al. [72] presented a brake anomaly detection system, which compares the brake data with the norm model to detect any vehicle misbehavior (e.g., due to software bugs or hardware glitches) in the Brake-by-Wire system. Other examples include utilizing fluid dynamics and electromagnetics as the basic laws to create prediction models for water system [56] and power grid [57], respectively. Based on the prediction models and predefined threat constraints, these methods check whether sensor readings are consistent with the expected behaviors of a control system. Cardenas et al. [4] proposed a physical model based detection method by monitoring the physical system under control, and the sensor and actuator values. The authors also proposed automatic response mechanisms by estimating the system states. Urbina et al. [55] discussed the limitations of existing physics-based attack detection approaches, i.e., they cannot limit the impact of stealthy attacks. The authors proposed a metric to measure the impact of stealthy attacks and to study the effectiveness of physics-based detection.

- Control policies. Physical model can also be specified by control policies. The main purpose of the policies is to improve the survivability of control systems, i.e., without losing critical functions under attacks. For example, McLaughlin et al. [76] introduced a policy enforcement for governing the usage of CPS devices, which checks whether the policy allows an operation depending on the state of the plant around the time the operation was issued. The policies specify what behaviors should be allowed to ensure the safety of physical machinery and assets.

- Cyber-physical model. Such a model captures the cyberphysical context dependency of control programs. Our proposed $e$ FSA characterizes control-program behaviors with respect to events, and enforces the runtime consistency among control decisions, values of data variables in control programs, and the physical environments. Thus, it is able to detect inconsistencies between the physical context and program execution.
As shown in Table 3 cyber models and physical models have different security guarantees. The former targets at detecting CPS control program anomalies in the cyber domain. While the latter mainly focuses on detecting false data injection attacks in the physical domain [57]. The cyber-physical interaction (i.e., interactions between cyber components and physical components) in CPS makes it challenging to predict runtime program behaviors through static analysis of the program code or model training. Existing cyber models [7], [31] are effective against control-oriented attacks, however, insufficient to detect data-oriented attacks. An effective CPS program anomaly detection needs to reason about program behaviors with respect to cyber-physical interactions, e.g., the decision of opening a valve has to be made based on the current water level of the tank. ContexIoT [30] provides context identification for sensitive actions in the permission granting process of IoT applications on Android platforms. Though both ContextIoT and $e$ FSA consider execution contextual integrity, ContextIoT does not support the detection of data-oriented attacks.

Distinctive from existing works in this area, our Orpheus focuses on utilizing the event-driven feature in control-program anomaly detection and our program behavior model combines both the cyber and physical aspects. Consequently, physics-based models, which can be inherently integrated into our approach to enhance security and efficiency, do not compete but rather complement our scheme. Stuxnet attack [8] manipulated the nuclear centrifuge's rotor speed, and fooled the system operator by replaying the recorded normal data stream during the attack [36]. Since $e$ FSA's detection is independent on the history data, it makes Stuxnet-like attacks detectable in $e$ FSA by detecting runtime inconsistencies between the physical context (runtime rotor speed) and the control program's behavior. In addition, attackers may exploit hardware vulnerabilities [79] to manipulate data in memory so as to launch attacks on control branch or control intensity. $e \mathrm{FSA}$ is also able to detect anomalies caused by such hardware attacks.

\section{CONCLUSION}

In this work, we presented Orpheus, a new security mechanism for CPS control programs in defending against data-oriented attacks, by enforcing cyber-physical execution semantics. As an FSAbased instantiation of Orpheus, we proposed the program behavior model $e$ FSA, which advances the state-of-the-art program behavior modelling. To the best of our knowledge, this is the first program behavior model that integrates both cyber and physical properties to defend against data-oriented attacks. We implemented a proof-of-concept prototype to demonstrate the feasibility of our approach. Real-world case studies demonstrated $e$ FSA's efficacy against different data-oriented attacks. As for our future work, we plan to integrate physics-based models into our approach, design robust event verification mechanisms, and extend the Orpheus design paradigm to support actuation integrity for fine-grained anomaly detection at the instruction level without the need of tracing facilities. We also plan to investigate the scalability of our approach on program size and complexity.

\section{ACKNOWLEDGMENTS}

This work has been supported by the Office of Naval Research under Grant ONR-N00014-17-1-2498, National Science Foundation under Grant OAC-1541105, and Security and Software Engineering Research Center (S2ERC), a NSF sponsored multi-university Industry/University Cooperative Research Center (I/UCRC). 


\section{References}

[1] L. Cheng, K. Tain, and D. D. Yao, "Enforcing cyber-physical execution semantics to defend against data-oriented attacks," in ACSAC, 2017.

[2] A. B. Sharma, F. Ivančić, A. Niculescu-Mizil, H. Chen, and G. Jiang, "Modeling and analytics for cyber-physical systems in the age of big data," SIGMETRICS Perform. Eval. Rev., vol. 41, no. 4, pp. 74-77, 2014.

[3] S. Sridhar, A. Hahn, and M. Govindarasu, "Cyber-physical system security for the electric power grid," Proceedings of the IEEE, vol. 100, no. 1 , pp. $210-224,2012$.

[4] A. A. Cárdenas, S. Amin, Z.-S. Lin, Y.-L. Huang, C.-Y. Huang, and S. Sastry, "Attacks against process control systems: Risk assessment, detection, and response," in ASIACCS, 2011.

[5] "Cyber-Physical Systems," www.cpse-labs.eu/cps.php [Accessed 09-122017].

[6] R. Mitchell and I.-R. Chen, "A survey of intrusion detection techniques for cyber-physical systems," ACM Comput. Surv., vol. 46, no. 4, pp. 55:1-55:29, Mar. 2014.

[7] T. Abera, N. Asokan, L. Davi, J. Ekberg, T. Nyman, A. Paverd, A. Sadeghi, and G. Tsudik, "C-FLAT: control-flow attestation for embedded systems software," in CCS, 2016.

[8] D. Kushner, "The real story of Stuxnet," IEEE Spectrum, vol. 50, no. 3, pp. $48-53,2013$.

[9] "The Industrial Control Systems Cyber Emergency Response Team," https://ics-cert.us-cert.gov/, [Accessed 09-12-2017].

[10] C. Zimmer, B. Bhat, F. Mueller, and S. Mohan, "Time-based intrusion detection in cyber-physical systems," in ICCPS, 2010.

[11] J. Habibi, A. Gupta, S. Carlsony, A. Panicker, and E. Bertino, "MAVR: Code reuse stealthy attacks and mitigation on unmanned aerial vehicles," in ICDCS, 2015, pp. 642-652.

[12] D. D. Chen, M. Egele, M. Woo, and D. Brumley, "Towards automated dynamic analysis for Linux-based embedded firmware," in NDSS, 2016.

[13] L. Garcia, F. Brasser, M. H. Cintuglu, A.-R. Sadeghi, O. Mohammed, and S. A. Zonouz, "Hey, my malware knows physics! attacking PLCs with physical model aware rootkit," in NDSS, 2017.

[14] T. Nyman, G. Dessouky, S. Zeitouni, A. Lehikoinen, A. Paverd, N. Asokan, and A. Sadeghi, "Hardscope: Thwarting DOP with hardwareassisted run-time scope enforcement," CoRR, vol. abs/1705.10295, 2017. [Online]. Available: http://arxiv.org/abs/1705.10295

[15] A. Francillon and C. Castelluccia, "Code injection attacks on Harvardarchitecture devices," in CCS, 2008.

[16] X. Shu, D. D. Yao, and B. G. Ryder, "A formal framework for program anomaly detection," in RAID, 2015.

[17] V. Kiriansky, D. Bruening, and S. P. Amarasinghe, "Secure execution via program shepherding," in USENIX Security, 2002.

[18] M. Abadi, M. Budiu, U. Erlingsson, and J. Ligatti, "Control-flow integrity," in CCS, 2005.

[19] A. Francillon, D. Perito, and C. Castelluccia, "Defending embedded systems against control flow attacks," in SecuCode, 2009.

[20] F. A. T. Abad, J. V. D. Woude, Y. Lu, S. Bak, M. Caccamo, L. Sha, R. Mancuso, and S. Mohan, "On-chip control flow integrity check for real time embedded systems," in 2013 IEEE 1st International Conference on Cyber-Physical Systems, Networks, and Applications, 2013.

[21] S. Lu and R. Lysecky, "Analysis of control flow events for timing-based runtime anomaly detection," in Proceedings of Workshop on Embedded Systems Security, 2015.

[22] N. Carlini, A. Barresi, M. Payer, D. Wagner, and T. R. Gross, "Controlflow bending: On the effectiveness of control-flow integrity," in USENIX Security, 2015.

[23] S. Chen, J. Xu, E. C. Sezer, P. Gauriar, and R. K. Iyer, "Non-control-data attacks are realistic threats," in USENIX Security, 2005.

[24] H. Hu, S. Shinde, S. Adrian, Z. L. Chua, P. Saxena, and Z. Liang, "Data-oriented programming: On the expressiveness of non-control data attacks," in IEEE $S \& P, 2016$.

[25] Y. Wang, Z. Xu, J. Zhang, L. Xu, H. Wang, and G. Gu, "Srid: State relation based intrusion detection for false data injection attacks in scada," in ESORICS, 2014.

[26] R. Tan, H. H. Nguyen, E. Y. S. Foo, X. Dong, D. K. Y. Yau, Z. Kalbarczyk, R. K. Iyer, and H. B. Gooi, "Optimal false data injection attack against automatic generation control in power grids," in ICCPS, 2016.

[27] S. Adepu, S. Shrivastava, and A. Mathur, "Argus: An orthogonal defense framework to protect public infrastructure against cyber-physical attacks," IEEE Internet Computing, vol. 20, no. 5, pp. 38-45, 2016.

[28] "German Steel Mill Meltdown," securityintelligence.com/ german-steel-mill-meltdown-rising-stakes-in-the-internet-of-things/ [Accessed 09-12-2017].

[29] P. Derler, E. A. Lee, S. Tripakis, and M. Törngren, "Cyber-physical system design contracts," in ICCPS, 2013.
[30] Y. J. Jia, Q. A. Chen, S. Wang, A. Rahmati, E. Fernandes, Z. M. Mao, and A. Prakash, "ContexIoT: Towards Providing Contextual Integrity to Appified IoT Platforms," in NDSS, 2017.

[31] M.-K. Yoon, S. Mohan, J. Choi, M. Christodorescu, and L. Sha, "Learning execution contexts from system call distribution for anomaly detection in smart embedded system," in Proceedings of the Second International Conference on Internet-of-Things Design and Implementation (IoTDI), 2017, pp. 191-196.

[32] C. Warrender, S. Forrest, and B. Pearlmutter, "Detecting intrusions using system calls: alternative data models," in IEEE S\&P, 1999.

[33] K. Xu, D. Yao, B. Ryder, and K. Tian, "Probabilistic program modeling for high-precision anomaly classification," in CSF, 2015.

[34] S. McLaughlin, D. Pohly, P. McDaniel, and S. Zonouz, "A trusted safety verifier for process controller code," in NDSS, 2014.

[35] J. Tan, H. J. Tay, U. Drolia, R. Gandhi, and P. Narasimhan, "PCFIRE: Towards provable preventative control-flow integrity enforcement for realistic embedded software," in 2016 International Conference on Embedded Software (EMSOFT), 2016, pp. 1-10.

[36] L. Garcia, F. Brasser, M. H. Cintuglu, A.-R. Sadeghi, O. Mohammed, and S. A. Zonouz, "Hey, My Malware Knows Physics! Attacking PLCs with Physical Model Aware Rootkit," in NDSS, 2017.

[37] M. Schwartz, J. Mulder, A. R. Chavez, and B. A. Allan, "Emerging techniques for field device security," IEEE Security and Privacy, vol. 12, no. 6, pp. 24-31, 2014.

[38] M. Darren, V. Fredrik, V. Giovanni, and K. Christopher, "Anomalous system call detection," ACM Trans. Inf. Syst. Secur, vol. 9, no. 1, pp. 61-93, 2006.

[39] X. Shu, D. Yao, and N. Ramakrishnan, "Unearthing stealthy program attacks buried in extremely long execution paths," in CCS, 2015.

[40] B. Ur, E. McManus, M. Pak Yong Ho, and M. L. Littman, "Practical trigger-action programming in the smart home," in CHI, 2014.

[41] A. A. Clements, N. S. Almakhdhub, K. S. Saab, P. Srivastava, J. Koo, S. Bagchi, and M. Payer, "Protecting bare-metal embedded systems with privilege overlays," in 2017 IEEE Symposium on Security and Privacy (SP), 2017, pp. 289-303.

[42] E. G. Chekole, S. Chattopadhyay, M. Ochoa, and G. Huaqun, "Enforcing full-stack memory-safety in cyber-physical systems," in Engineering Secure Software and Systems, M. Payer, A. Rashid, and J. M. Such, Eds. Springer International Publishing, 2018, pp. 9-26.

[43] Z. Sun, B. Feng, L. Lu, and S. Jha, "OEI: operation execution integrity for embedded devices," CoRR, vol. abs/1802.03462, 2018. [Online]. Available: http://arxiv.org/abs/1802.03462

[44] R. Sekar, M. Bendre, D. Dhurjati, and P. Bollineni, "A fast automatonbased method for detecting anomalous program behaviors," in IEEE $S \& P, 2001$.

[45] X. Shu, D. D. Yao, N. Ramakrishnan, and T. Jaeger, "Long-span program behavior modeling and attack detection," ACM Transactions on Privacy and Security, vol. 20, no. 4, pp. 1-28, Sep. 2017.

[46] D. Wagner and P. Soto, "Mimicry attacks on host-based intrusion detection systems," in CCS, 2002.

[47] “LLVM," http://llvm.org/, [Accessed 09-12-2017].

[48] E. J. Schwartz, T. Avgerinos, and D. Brumley, "All you ever wanted to know about dynamic taint analysis and forward symbolic execution (but might have been afraid to ask)," in IEEE $S \& P, 2010$.

[49] "GPIO access library for RPI," wiringpi.com/, [Accessed 09-12-2017]

[50] X. Zhang, R. Gupta, and Y. Zhang, "Precise dynamic slicing algorithms," in ICSE' $03,2003$.

[51] D. Gao, M. K. Reiter, and D. Song, "On gray-box program tracking for anomaly detection," in USENIX Security, 2004.

[52] D. Wagner and D. Dean, "Intrusion detection via static analysis," in IEEE $S \& P, 2001$.

[53] L. C. Lam and C. Tzi-cker, "Automatic extraction of accurate applicationspecific sandboxing policy," in RAID, 2004.

[54] “Arduino," www.arduino.cc/, [Accessed 09-12-2017].

[55] D. I. Urbina, J. A. Giraldo, A. A. Cardenas, N. O. Tippenhauer, J. Valente, M. Faisal, J. Ruths, R. Candell, and H. Sandberg, "Limiting the impact of stealthy attacks on industrial control systems," in CCS, 2016.

[56] D. Hadžiosmanović, R. Sommer, E. Zambon, and P. H. Hartel, "Through the eye of the PLC: Semantic security monitoring for industrial processes," in ACSAC, 2014.

[57] Y. Liu, P. Ning, and M. K. Reiter, "False data injection attacks against state estimation in electric power grids," in CCS, 2009.

[58] "Software-artifact Infrastructure Repository," http://sir.unl.edu/, [Accessed 09-12-2017].

[59] M. Bahrepour, N. Meratnia, and P. J. M. Havinga, "Sensor fusion-based event detection in wireless sensor networks," in Annual International 
Mobile and Ubiquitous Systems: Networking Services, MobiQuitous, 2009.

[60] Y. Zhuang, E. Gessiou, S. Portzer, F. Fund, M. Muhammad, I. Beschastnikh, and J. Cappos, "Netcheck: Network diagnoses from blackbox traces," in USENIX NSDI, 2014, pp. 115-128.

[61] N. M. Rodday, R. d. O. Schmidt, and A. Pras, "Exploring security vulnerabilities of unmanned aerial vehicles," in NOMS, 2016.

[62] "Opto 22 connects real-world industrial devices to millions of Raspberry Pi,” www.prweb.com/releases/2016/11/prweb13853953.htm, [Accessed 09-12-2017]

[63] "The REX Control System for Raspberry Pi," www.rexcontrols.com/, [Accessed 09-12-2017].

[64] H. Feng, J. Giffin, Y. Huang, S. Jha, W. Lee, and B. Miller, "Formalizing sensitivity in static analysis for intrusion detection," in IEEE S\&P, 2004.

[65] V. Adrien, E.-J. Naser, and D. M. R., "Hardware-assisted software event tracing," Concurrency and Computation: Practice and Experience, 2017.

[66] Z. Ning and F. Zhang, "Ninja: Towards transparent tracing and debugging on arm," in USENIX Security, 2017.

[67] A. Munir and F. Koushanfar, "Design and analysis of secure and dependable automotive cps: A steer-by-wire case study," IEEE Transactions on Dependable and Secure Computing, 2018.

[68] "ZingBox: Enabling the Internet of Trusted Things," www.zingbox.com/, [Accessed 09-12-2017].

[69] J. Valente, C. Barreto, and A. A. Cárdenas, "Cyber-physical systems attestation," in DCOSS, 2014.

[70] R. Mitchell and I.-R. Chen, "Adaptive intrusion detection of malicious unmanned air vehicles using behavior rule specifications," IEEE Transactions on Systems, Man, and Cybernetics: Systems, vol. 44, no. 5, pp. 593-604, 2014.

[71] R. Mitchell and I. R. Chen, "Behavior rule specification-based intrusion detection for safety critical medical cyber physical systems," IEEE Transactions on Dependable and Secure Computing, vol. 12, no. 1, pp. 16-30, 2015.

[72] K.-T. Cho, K. G. Shin, and T. Park, "CPS approach to checking norm operation of a brake-by-wire system," in ICCPS, 2015.

[73] I. Rouf, R. Miller, H. Mustafa, T. Taylor, S. Oh, W. Xu, M. Gruteser, W. Trappe, and I. Seskar, "Security and privacy vulnerabilities of incar wireless networks: A tire pressure monitoring system case study," in USENIX Security, 2010.

[74] C. Feng, T. Li, and D. Chana, "Multi-level anomaly detection in industrial control systems via package signatures and LSTM networks," in DSN, 2017.

[75] C. M. Ahmed, M. Ochoa, J. Zhou, A. P. Mathur, R. Qadeer, C. Murguia, and J. Ruths, "Noiseprint: Attack detection using sensor and process noise fingerprint in cyber physical systems," in Proceedings of the 2018 on Asia Conference on Computer and Communications Security, ser. ASIACCS '18, 2018, pp. 483-497.

[76] S. McLaughlin, "CPS: Stateful policy enforcement for control system device usage," in ACSAC, 2013, pp. 109-118.

[77] S. Mohan, S. Bak, E. Betti, H. Yun, L. Sha, and M. Caccamo, "S3A: Secure system simplex architecture for enhanced security and robustness of cyber-physical systems," in $\mathrm{HiCoNS}, 2013$.

[78] M.-K. Yoon, S. Mohan, J. Choi, J.-E. Kim, and L. Sha, "Securecore: A multicore-based intrusion detection architecture for real-time embedded systems," in RTAS, 2013.

[79] V. van der Veen, Y. Fratantonio, M. Lindorfer, D. Gruss, C. Maurice, G. Vigna, H. Bos, K. Razavi, and C. Giuffrida, "Drammer: Deterministic rowhammer attacks on mobile platforms," in CCS '16, 2016, pp. 16751689.

Long Cheng is currently an assistant professor of computer science at Clemson University, USA. He received his second PhD in Computer Science from Virginia Tech USA in 2018, and the first PhD from Beijing University of Posts and Telecommunications China in 2012. He worked as a Research Scientist at the Institute for Infocomm Research $\left(I^{2} R\right)$, Singapore from 2014 to 2015, and a Research Fellow at Singapore University of Technology and Design from 2012 to 2014. His research interests include system and network security, cyber forensics, cyberphysical systems (CPS) security, Internet of Things, mobile computing, and wireless networks. He received the Best Paper Award from IEEE Wireless Communications and Networking Conference (WCNC) in 2013, the Erasmus Mundus Scholar Award in 2014, and the Pratt Fellowship at Virginia Tech in 2017.
Ke Tian is currently a data scientist at Microsoft. His experience is on leveraging Machine Learning and Anomaly Detection to address cyber security challenges. He received his $\mathrm{PhD}$ degree from the Department of Computer Science at Virginia Tech in 2018. He received his bachelor degree majoring information security from University of Science and Technology of China in 2013. His research interests are in cybersecurity, mobile security and machine learning.

Danfeng (Daphne) Yao is an associate professor of computer science at Virginia Tech. In the past decade, she has been working on designing and developing data-driven anomaly detection techniques for securing networked systems against stealthy exploits and attacks. Her expertise also includes mobile security. Dr. Yao received her Ph.D. in Computer Science from Brown University. Dr. Yao is an Elizabeth and James E. Turner Jr. '56 Faculty Fellow and L-3 Faculty Fellow. She received the NSF CAREER Award in 2010 for her work on human-behavior driven malware detection, and the ARO Young Investigator Award for her semantic reasoning for mission-oriented security work in 2014 . She has several Best Paper Awards (e.g., ICNP '12, CollaborateCom '09, and ICICS '06) and Best Poster Awards (e.g., ACM CODASPY '15). She was given the Award for Technological Innovation from Brown University in 2006. She held multiple U.S. patents for her anomaly detection technologies. Dr. Yao is an associate editor of IEEE Transactions on Dependable and Secure Computing (TDSC). She serves as PC members in numerous computer security conferences, including ACM CCS and IEEE S\&P. She has over 85 peer-reviewed publications in major security and privacy conferences and journals.

Lui Sha received the Ph.D. degree in computer science from Carnegie Mellon University, Pittsburgh, PA, USA, in 1985. He is currently a Donald B. Gillies Chair Professor of Computer Science with the University of Illinois at Urbana Champaign, Champaign, IL, USA. His work on realtime computing is supported by most of the open standards in realtime computing and has been cited as a key element in the success of many national high-technology projects including GPS upgrade, the Mars Pathfinder, and the International Space Station.

Raheem Beyah is the Motorola Foundation Professor and Associate Chair for Strategic Initiatives and Innovation in the School of Electrical and Computer Engineering at Georgia Tech, where he leads the Communications Assurance and Performance Group (CAP). His research interests include network security, wireless networks, network traffic characterization and performance, and security visualization. $\mathrm{He}$ received the National Science Foundation CAREER award in 2009 and was selected for DARPA's Computer Science Study Panel in 2010. He is a member of AAAS, ASEE, a lifetime member of NSBE, a senior member of IEEE, and an ACM Distinguished Scientist. 